\title{
Temperature-Dependent Functional Response of Harmonia Axyridis Pallas (Coleoptera: Coccinellidae) Preying on Acyrthosiphon Pisum (Harris) (Hemiptera: Aphididae) Nymphs
}

\author{
Yasir Islam \\ Huazhong Agricultural University \\ Farhan Mahmood Shah \\ Bahauddin Zakariya University \\ Xu Rubing \\ Tobacco Research Institute \\ Muhammad Razaq \\ Bahauddin Zakariya University \\ Miao Yabo \\ Huazhong Agricultural University \\ Li Xihong
}

Tobacco Research Institute

Xingmiao Zhou ( $\boldsymbol{\nabla}$ xmzhou@mail.hzau.edu.cn )

Huazhong Agricultural University

\section{Research Article}

Keywords: Aphid, Coccinellid, Attack rate, Handling time, Predation, Global warming

Posted Date: April 21st, 2021

DOI: https://doi.org/10.21203/rs.3.rs-439244/v1

License: (a) (i) This work is licensed under a Creative Commons Attribution 4.0 International License. Read Full License 


\section{Abstract}

Functional response models are often used to understand the foraging interactions and determine the suitable biocontrol agents. We determined the functional response of Harmonia axyridis to nymph Acyrthosiphon pisum at different but constant temperatures (between 15 and $35^{\circ} \mathrm{C}$ ) and prey densities. Logistic regression and Roger's random predator models were employed to determine the type and parameters of functional response. Harmonia axyridis larvae and adults exhibited Type II functional responses to different densities of $A$. pisum. Warming increased both the predation activity and host aphid control mortality. The $4^{\text {th }}$ instar and female $H$. axyridis consumed the most aphids. Warming contributed markedly in accelerating the predator action. For fourth instar larvae and female $H$. axyridis adult, the successful attack rates were $0.234 \pm 0.014 \mathrm{~h}^{-1}$ and $0.247 \pm 0.015 \mathrm{~h}^{-1}$; the handling times were $0.132 \pm 0.005 \mathrm{~h}$ and $0.156 \pm 0.004 \mathrm{~h}$; and the estimated maximum predation rates were $181.28 \pm 14.54$ and $153.85 \pm 4.06$, respectively. These findings accentuate the high performance of $4^{\text {th }}$ instar and female $H$. axyridis and the role of temperature in their efficiency. Further studies exploring intraguild predation and mutual interference will be required to conclude the biocontrol potential of $H$. axyridis to $A$. pisum.

\section{Introduction}

The concept of integrated pest management strategies based on biocontrol agents has received increasing prominence worldwide ${ }^{1}$. It has been implemented with tremendous success to both under fields and greenhouses ${ }^{2-4}$, in the particular context of reducing the large-scale use of pesticides ${ }^{5-8}$. While the adoption of biological control is desirable, the successful implementation depends upon the comprehensive understanding of predator-prey interactions, owing to their fundamental role towards ecosystem functionality and food web stability ${ }^{9}$. Several methods can be applied to quantifying these interactions ${ }^{10,11}$, functional response ${ }^{12}$, numerical response ${ }^{13}$, kill rate ${ }^{14}$, and consumption rate ${ }^{15}$ are typically employed when foraging interactions have to formalize ${ }^{16}$. Functional response describes how predation rate changes with resource density ${ }^{17}$. Three types of functional responses can be present, with consumption rate being linear up to a constant plateau (Type I), hyperbolic (Type II), or sigmoid (Type III) ${ }^{18}$, depending upon the parameter of functional response, i.e., the enemy attack rate, and prey handling time. The attack rate defines the steepness of the increase in predation with the increase of prey density, and handling time helps rate the satiation threshold ${ }^{19}$. Many sources of variation are known to modulate outcomes of these functional response parameters ${ }^{20,21}$.

Temperature is a chief driver of biological systems through the temperature-dependent nature of biological rates (e.g., metabolic rates) ${ }^{24}$. Effects of temperature on biological rates are likely to be realized up to species level thus expected to influence the population growth rates and carrying capacities ${ }^{25}$ as well as ecosystem functions. Temperature can regulate the prey-predator interactions by altering their responses to metamorphosis and population dynamics ${ }^{22}$. Warming is shown to increase the food intake 
of predators ${ }^{23}$. In order to consume large prey biomass, a predator should become quickly adept to search/handle its prey, so that it may spend time on consumption events rather than on prey searching/handling attempts, therefore a change of predatory behavior or functional response may be expected under warming ${ }^{26,27}$. For instance, the handling time decreased, consumption rate increased, and the type of functional response changed from Type II to Type III for Podisus maculiventris (Say) and Podisus nigrispinus (Dallas) (Hemiptera: Pentatomidae) preying on Spodoptera exigua (Hübner) (Lepidoptera: Noctuidae) when the temperature increased from $18^{\circ} \mathrm{C}$ to $27^{\circ} \mathrm{C}^{28}$. Similarly, functional response for Euborellia annulipes (Dermaptera: Anisolabididae) preying on larvae Plutella xylostella (Lepidoptera: Plutellidae) changed from Type III at low temperature (i.e., $18^{\circ} \mathrm{C}$ ) to Type II at higher temperature (i.e., $25^{\circ} \mathrm{C}$ and $\left.32^{\circ} \mathrm{C}\right)^{29}$. A changing functional response with warming may destabilize some predator-prey interactions, it may favor prey depletion by increasing predator action ${ }^{29}$. Therefore, a large body of literature is directed towards understanding the consequence of warming on food webs and stability $^{30,31}$.

The pea aphid, Acyrthosiphon pisum (Harris) (Hemiptera: Aphididae), originally a Palearctic species ${ }^{32}$, has now become a pest of global concern for pulse and legume producers ${ }^{33}$. It has a broad host range, infesting grass pea (Lathryus sativus L.), faba bean (Vicia faba L.), pea (Pisum sativum L.), alfalfa (Medicago sativa L.), chickpea (Cicer arietinum L.), lentil (Lens culinaris Medik.), and lupin (Lupinus albus L.) (Fabales: Fabaceae) ${ }^{34}$. The aphid inflicts injury either directly, i.e., by removing sap from succulent phloem tissues or via injecting phytotoxic saliva ${ }^{35}$, or indirectly, i.e., by vectoring multiple plant viruses (e.g., the cucumber mosaic virus, the pea enation mosaic virus, the bean leaf roll virus, and the beet yellow virus) ${ }^{36,37}$ or by producing honeydew, subsequently inviting sooty-mould that disturbs plant photosynthetic and respirational functions ${ }^{38}$. Its prolonged infestation could lead to plant stunting, deformation, and discoloration, ultimately reducing crop yields by $35.7 \% 39$. The broad host range, complex life cycle, and quick adaptiveness to new environments make it difficult to control this aphid. Moreover, this aphid has been reported for the development of insecticide resistance ${ }^{40}$.

Many aphidophagous ladybird beetles (Coleoptera: Coccinellidae) are known to be exploited for conservative or augmentative biocontrol programs of several economically important aphids in diverse crops, outdoor and in greenhouses, allowing aphid suppression to well below economically damaging levels ${ }^{41}$. Harmonia axyridis Pallas (Coleoptera: Coccinellidae) is a generalist predator, geographically wide-spread ${ }^{42}$, and extensively employed as a biocontrol agent of soft-bodied insects, including aphids in a diversity of crops ${ }^{43,44}$. Various biological aspects of $H$. axyridis of importance for its predatory potential (e.g. phenological characteristics, life table parameters, and functional response (generally with a Type II response), have been investigated with respect to the temperature and other concerning factors ${ }^{45-49}$ to many crop pests ${ }^{50-52}$, however, information on its predatory behavior and functional response to $A$. pisum almost lacking ${ }^{53}$. 
Here, we report the functional response of $H$. axyridis to $A$. pisum under various thermal conditions. We expect that, based on its close association with predator growth and development, the temperature change will accordingly modify consumption. Further, we aim to assess whether thermal conditions and aphid density affect the functional response of larvae and adult $H$. axyridis.

\section{Results}

The mortalities of host aphid in the controls were different between temperature $\left(F_{4,160}=78.64 ; P<\right.$ 0.001 ; Fig. 1a) and aphid density $\left(F_{7,160}=116.75 ; P<0.001\right.$; Fig. $\left.1 \mathrm{~b}\right)$, each increased with increasing the temperature and host aphid density. The density-wise assessment showed similar behavior for increasing trajectories of host mortalities with warming and increasing aphid density $\left(F_{28,160}=10.23 ; P<0.001\right.$; Fig. 1c).

Harmonia axyridis adults (female: $30.21 \pm 5.12$ aphids/day; male: $23.45 \pm 3.71$ aphids/day) and 4 th instar (32.25 \pm 5.27 aphids/day) consumed significantly more aphids than preceded instars (Wald $X^{2}=$ 539.39; $\mathrm{df}=5 ; P<0.001$; Fig. 2b), with an exponentially increasing consumption with respect to increasing temperature (Wald $X^{2}=104.86$; $\mathrm{df}=4 ; P<0.001$; Fig. 2a) and aphid density (Wald $X^{2}=1759.52 ; \mathrm{df}=7 ; P<$ 0.001 ; Fig. $2 \mathrm{c}$ ), peaking at $30^{\circ} \mathrm{C}$ (i.e., $24.41 \pm 6.11$ aphids/day) and $35^{\circ} \mathrm{C}$ (i.e., $27.03 \pm 6.43$ aphids/day), at aphid densities of 128 and 160 aphids/petri dish arena. Three way (temperature $\times$ stage $\times$ density: Wald $X^{2}=15.07 ; \mathrm{df}=140 ; P=1$ ) and two way (temperature $\times$ stage: Wald $X^{2}=27.78 ; \mathrm{df}=20 ; P=0.115$; temperature $\times$ density: Wald $\left.X^{2}=20.24 ; \mathrm{df}=28 ; P=0.855\right)$ interactions were non-significant, meaning consumption outcomes for temperature (Fig. 3) and growth stage (Fig. 4) remained unchanged, when evaluated at various aphid densities, separately.

Logistic regression between the initial aphid densities offered to larvae and adult $H$. axyridis and the proportion of aphid consumed ( $\mathrm{Na} / \mathrm{No}$ ) showed all significantly negative values of the linear coefficients $P_{1}$; exhibiting a Type II functional response across all growth stages and temperatures tested (Table 1). The declining consumption with increasing aphid densities (Fig. 5) also confirmed a Type Il functional response. The monotonically declining proportion of consumption with increased aphid densities, led to further confirmation of Type II functional responses (Fig. 6). Clearly, a more linear trend for the proportion of prey eaten with increasing aphid density was noted for 4th instar (Fig. 6d) and female H. axyridis (Fig. 6f) at higher (i.e., 30 and $35^{\circ} \mathrm{C}$ ) than lower temperatures. 
Table 1

Results of the logistic regression analysis of the proportion of nymphs of Acyrthosiphon pisum predated by all stages of Harmonia axyridis relative to the initial number of nymphs provided.

\begin{tabular}{|c|c|c|c|c|c|c|}
\hline Temperatures & $\begin{array}{l}\text { Growth } \\
\text { Stages }\end{array}$ & Parameters & Estimates & S.E. & $\begin{array}{l}Z- \\
\text { Value }\end{array}$ & $\operatorname{Pr}(\mathrm{z})$ \\
\hline \multirow[t]{14}{*}{$15^{\circ} \mathrm{C}$} & 1st instar & Intercept & $\begin{array}{l}-7.39 \times 10^{-} \\
01\end{array}$ & $\begin{array}{l}2.26 \times 10^{-} \\
01\end{array}$ & -3.26 & 0.001 \\
\hline & & Linear & $\begin{array}{l}-6.96 \times 10^{-} \\
02\end{array}$ & $\begin{array}{l}1.37 \times 10^{-} \\
02\end{array}$ & -5.05 & $\begin{array}{l}4.36 \times 10^{-} \\
07\end{array}$ \\
\hline & & Quadratic & $\begin{array}{l}6.449 \times 10^{-} \\
04\end{array}$ & $\begin{array}{l}1.962 \times 10^{-} \\
04\end{array}$ & 3.286 & $\begin{array}{l}1.01 \times 10^{-} \\
03\end{array}$ \\
\hline & & Cubic & $\begin{array}{l}-2.040 \times 10^{-} \\
06\end{array}$ & $\begin{array}{l}7.740 \times 10^{-} \\
07\end{array}$ & -2.635 & $\begin{array}{l}8.40 \times 10^{-} \\
03\end{array}$ \\
\hline & 2nd instar & Intercept & $\begin{array}{l}-3.67 \times 10^{-} \\
02\end{array}$ & $\begin{array}{l}2.03 \times 10^{-} \\
01\end{array}$ & -0.18 & 0.85 \\
\hline & & Linear & $\begin{array}{l}-8.96 \times 10^{-} \\
02\end{array}$ & $\begin{array}{l}1.25 \times 10^{-} \\
02\end{array}$ & -7.15 & $\begin{array}{l}8.15 \times 10^{-} \\
13\end{array}$ \\
\hline & & Quadratic & $\begin{array}{l}8.635 \times 10^{-} \\
04\end{array}$ & $\begin{array}{l}1.771 \times 10^{-} \\
04\end{array}$ & 4.876 & $\begin{array}{l}1.08 \times 10^{-} \\
06\end{array}$ \\
\hline & & Cubic & $\begin{array}{l}-2.73 \times 10^{-} \\
06\end{array}$ & $\begin{array}{l}6.95 \times 10^{-} \\
07\end{array}$ & -3.941 & $\begin{array}{l}8.12 \times 10^{-} \\
05\end{array}$ \\
\hline & 3rd instar & Intercept & 1.58 & $\begin{array}{l}1.93 \times 10^{-} \\
01\end{array}$ & 8.19 & $\begin{array}{l}2.42 \times 10^{-} \\
16\end{array}$ \\
\hline & & Linear & $\begin{array}{l}-1.06 \times 10^{-} \\
01\end{array}$ & $\begin{array}{l}9.99 \times 10^{-} \\
03\end{array}$ & -10.67 & $<2 \times 10^{-16}$ \\
\hline & & Quadratic & $\begin{array}{l}1.03 \times 10^{-} \\
03\end{array}$ & $\begin{array}{l}1.30 \times 10^{-} \\
04\end{array}$ & 7.887 & $\begin{array}{l}3.09 \times 10^{-} \\
15\end{array}$ \\
\hline & & Cubic & $\begin{array}{l}-3.24 \times 10^{-} \\
06\end{array}$ & $\begin{array}{l}4.89 \times 10^{-} \\
07\end{array}$ & -6.624 & $\begin{array}{l}3.50 \times 10^{-} \\
11\end{array}$ \\
\hline & 4th instar & Intercept & 3.35 & $\begin{array}{l}2.62 \times 10^{-} \\
01\end{array}$ & 12.76 & $<2 \times 10^{-16}$ \\
\hline & & Linear & $\begin{array}{l}-1.18 \times 10^{-} \\
01\end{array}$ & $\begin{array}{l}1.08 \times 10^{-} \\
02\end{array}$ & -10.93 & $<2 \times 10^{-16}$ \\
\hline
\end{tabular}




\begin{tabular}{|c|c|c|c|c|c|c|}
\hline \multirow[t]{11}{*}{ Temperatures } & $\begin{array}{l}\text { Growth } \\
\text { Stages }\end{array}$ & Parameters & Estimates & S.E. & $\begin{array}{l}Z- \\
\text { Value }\end{array}$ & $\operatorname{Pr}(\mathrm{z})$ \\
\hline & & Quadratic & $\begin{array}{l}1.08 \times 10^{-} \\
03\end{array}$ & $\begin{array}{l}1.26 \times 10^{-} \\
04\end{array}$ & 8.580 & $<2 \times 10^{-16}$ \\
\hline & & Cubic & $\begin{array}{l}-3.30 \times 10^{-} \\
06\end{array}$ & $\begin{array}{l}4.43 \times 10^{-} \\
07\end{array}$ & -7.462 & $\begin{array}{l}8.52 \times 10^{-} \\
14\end{array}$ \\
\hline & Male & Intercept & 2.30 & $\begin{array}{l}2.13 \times 10^{-} \\
01\end{array}$ & 10.77 & $<2 \times 10^{-16}$ \\
\hline & & Linear & $\begin{array}{l}-9.59 \times 10^{-} \\
02\end{array}$ & $\begin{array}{l}9.68 \times 10^{-} \\
03\end{array}$ & -9.91 & $<2 \times 10^{-16}$ \\
\hline & & Quadratic & $\begin{array}{l}8.61 \times 10^{-} \\
04\end{array}$ & $\begin{array}{l}1.20 \times 10^{-} \\
04\end{array}$ & 7.176 & $\begin{array}{l}7.20 \times 10^{-} \\
13\end{array}$ \\
\hline & & Cubic & $\begin{array}{l}-2.62 \times 10^{-} \\
06\end{array}$ & $\begin{array}{l}4.35 \times 10^{-} \\
07\end{array}$ & -6.012 & $\begin{array}{l}1.84 \times 10^{-} \\
09\end{array}$ \\
\hline & Female & Intercept & 2.47 & $\begin{array}{l}2.19 \times 10^{-} \\
01\end{array}$ & 11.27 & $<2 \times 10^{-16}$ \\
\hline & & Linear & $\begin{array}{l}-9.75 \times 10^{-} \\
02\end{array}$ & $\begin{array}{l}9.60 \times 10^{-} \\
03\end{array}$ & -10.15 & $<2 \times 10^{-16}$ \\
\hline & & Quadratic & $\begin{array}{l}9.13 \times 10^{-} \\
04\end{array}$ & $\begin{array}{l}1.16 \times 10^{-} \\
04\end{array}$ & 7.877 & $\begin{array}{l}3.36 \times 10^{-} \\
15\end{array}$ \\
\hline & & Cubic & $\begin{array}{l}-2.81 \times 10^{-} \\
06\end{array}$ & $\begin{array}{l}4.13 \times 10^{-} \\
07\end{array}$ & -6.808 & $\begin{array}{l}9.90 \times 10^{-} \\
12\end{array}$ \\
\hline \multirow[t]{5}{*}{$20^{\circ} \mathrm{C}$} & 1st instar & Intercept & $\begin{array}{l}-8.50 \times 10^{-} \\
02\end{array}$ & $\begin{array}{l}1.95 \times 10^{-} \\
01\end{array}$ & -0.43 & 0.66 \\
\hline & & Linear & $\begin{array}{l}-7.39 \times 10^{-} \\
02\end{array}$ & $\begin{array}{l}1.12 \times 10^{-} \\
02\end{array}$ & -6.55 & $\begin{array}{l}5.42 \times 10^{-} \\
11\end{array}$ \\
\hline & & Quadratic & $\begin{array}{l}7.037 \times 10^{-} \\
04\end{array}$ & $\begin{array}{l}1.561 \times 10^{-} \\
04\end{array}$ & 4.509 & $\begin{array}{l}6.52 \times 10^{-} \\
06\end{array}$ \\
\hline & & Cubic & $\begin{array}{l}-2.237 \times 10^{-} \\
06\end{array}$ & $\begin{array}{l}6.045 \times 10^{-} \\
07\end{array}$ & -3.700 & $\begin{array}{l}2.16 \times 10^{-} \\
04\end{array}$ \\
\hline & 2nd instar & Intercept & $2.97 \times 10^{-}$ & $\begin{array}{l}1.92 \times 10^{-} \\
01\end{array}$ & 0.155 & 0.87 \\
\hline
\end{tabular}




\begin{tabular}{|c|c|c|c|c|c|c|}
\hline Temperatures & $\begin{array}{l}\text { Growth } \\
\text { Stages }\end{array}$ & Parameters & Estimates & S.E. & $\begin{array}{l}Z- \\
\text { Value }\end{array}$ & $\operatorname{Pr}(\mathrm{z})$ \\
\hline & & Linear & $\begin{array}{l}-7.59 \times 10^{-} \\
02\end{array}$ & $\begin{array}{l}1.09 \times 10^{-} \\
02\end{array}$ & -6.92 & $\begin{array}{l}4.32 \times 10^{-} \\
12\end{array}$ \\
\hline & & Quadratic & $\begin{array}{l}7.33 \times 10^{-} \\
04\end{array}$ & $\begin{array}{l}1.50 \times 10^{-} \\
04\end{array}$ & 4.871 & $\begin{array}{l}1.11 \times 10^{-} \\
06\end{array}$ \\
\hline & & Cubic & $\begin{array}{l}-2.33 \times 10^{-} \\
06\end{array}$ & $\begin{array}{l}5.79 \times 10^{-} \\
07\end{array}$ & -4.031 & $\begin{array}{l}5.56 \times 10^{-} \\
05\end{array}$ \\
\hline & 3rd instar & Intercept & 2.01 & $\begin{array}{l}2.03 \times 10^{-} \\
01\end{array}$ & 9.92 & $<2 \times 10^{-16}$ \\
\hline & & Linear & $\begin{array}{l}-9.10 \times 10^{-} \\
02\end{array}$ & $\begin{array}{l}9.43 \times 10^{-} \\
03\end{array}$ & -9.64 & $<2 \times 10^{-16}$ \\
\hline & & Quadratic & $\begin{array}{l}8.24 \times 10^{-} \\
04\end{array}$ & $\begin{array}{l}1.18 \times 10^{-} \\
04\end{array}$ & 6.959 & $\begin{array}{l}3.43 \times 10^{-} \\
12\end{array}$ \\
\hline & & Cubic & $\begin{array}{l}-2.53 \times 10^{-} \\
06\end{array}$ & $\begin{array}{l}4.33 \times 10^{-} \\
07\end{array}$ & -5.838 & $\begin{array}{l}5.29 \times 10^{-} \\
09\end{array}$ \\
\hline & 4th instar & Intercept & 3.82 & $\begin{array}{l}3.08 \times 10^{-} \\
01\end{array}$ & 12.41 & $<2 \times 10^{-16}$ \\
\hline & & Linear & $\begin{array}{l}-1.09 \times 10^{-} \\
01\end{array}$ & $\begin{array}{l}1.18 \times 10^{-} \\
02\end{array}$ & -9.25 & $<2 \times 10^{-16}$ \\
\hline & & Quadratic & $\begin{array}{l}9.71 \times 10^{-} \\
04\end{array}$ & $\begin{array}{l}1.32 \times 10^{-} \\
04\end{array}$ & 7.337 & $\begin{array}{l}2.19 \times 10^{-} \\
13\end{array}$ \\
\hline & & Cubic & $\begin{array}{l}-2.90 \times 10^{-} \\
06\end{array}$ & $\begin{array}{l}4.48 \times 10^{-} \\
07\end{array}$ & -6.471 & $\begin{array}{l}9.74 \times 10^{-} \\
11\end{array}$ \\
\hline & Male & Intercept & 2.85 & $\begin{array}{l}2.36 \times 10^{-} \\
01\end{array}$ & 12.04 & $<2 \times 10^{-16}$ \\
\hline & & Linear & $\begin{array}{l}-1.06 \times 10^{-} \\
01\end{array}$ & $\begin{array}{l}1.00 \times 10^{-} \\
02\end{array}$ & -10.51 & $<2 \times 10^{-16}$ \\
\hline & & Quadratic & $\begin{array}{l}9.86 \times 10^{-} \\
04\end{array}$ & $\begin{array}{l}1.19 \times 10^{-} \\
04\end{array}$ & 8.232 & $<2 \times 10^{-16}$ \\
\hline & & Cubic & $\begin{array}{l}-3.02 \times 10^{-} \\
06\end{array}$ & $\begin{array}{l}4.22 \times 10^{-} \\
07\end{array}$ & -7.152 & $\begin{array}{l}8.54 \times 10^{-} \\
13\end{array}$ \\
\hline
\end{tabular}




\begin{tabular}{|c|c|c|c|c|c|c|}
\hline Temperatures & $\begin{array}{l}\text { Growth } \\
\text { Stages }\end{array}$ & Parameters & Estimates & S.E. & $\begin{array}{l}Z- \\
\text { Value }\end{array}$ & $\operatorname{Pr}(\mathrm{z})$ \\
\hline & Female & Intercept & 2.68 & $\begin{array}{l}2.36 \times 10^{-} \\
01\end{array}$ & 11.39 & $<2 \times 10^{-16}$ \\
\hline & & Linear & $\begin{array}{l}-8.48 \times 10^{-} \\
02\end{array}$ & $\begin{array}{l}9.81 \times 10^{-} \\
03\end{array}$ & -8.64 & $<2 \times 10^{-16}$ \\
\hline & & Quadratic & $\begin{array}{l}7.68 \times 10^{-} \\
04\end{array}$ & $\begin{array}{l}1.15 \times 10^{-} \\
04\end{array}$ & 6.661 & $\begin{array}{l}2.72 \times 10^{-} \\
11\end{array}$ \\
\hline & & Cubic & $\begin{array}{l}-2.35 \times 10^{-} \\
06\end{array}$ & $\begin{array}{l}4.04 \times 10^{-} \\
07\end{array}$ & -5.827 & $\begin{array}{l}5.65 \times 10^{-} \\
09\end{array}$ \\
\hline \multirow[t]{11}{*}{$25^{\circ} \mathrm{C}$} & 1st instar & Intercept & $\begin{array}{l}4.89 \times 10^{-} \\
01\end{array}$ & $\begin{array}{l}1.87 \times 10^{-} \\
01\end{array}$ & 2.61 & 0.009 \\
\hline & & Linear & $\begin{array}{l}-8.64 \times 10^{-} \\
02\end{array}$ & $\begin{array}{l}1.06 \times 10^{-} \\
02\end{array}$ & -8.12 & $\begin{array}{l}4.54 \times 10^{-} \\
16\end{array}$ \\
\hline & & Quadratic & $\begin{array}{l}8.267 \times 10^{-} \\
04\end{array}$ & $\begin{array}{l}1.463 \times 10^{-} \\
04\end{array}$ & 5.652 & $\begin{array}{l}1.59 \times 10^{-} \\
08\end{array}$ \\
\hline & & Cubic & $\begin{array}{l}-2.619 \times 10^{-} \\
06\end{array}$ & $\begin{array}{l}5.645 \times 10^{-} \\
07\end{array}$ & -4.639 & $\begin{array}{l}3.51 \times 10^{-} \\
06\end{array}$ \\
\hline & 2nd instar & Intercept & $\begin{array}{l}4.75 \times 10^{-} \\
01\end{array}$ & $\begin{array}{l}1.78 \times 10^{-} \\
01\end{array}$ & 2.65 & 0.007 \\
\hline & & Linear & $\begin{array}{l}-6.27 \times 10^{-} \\
02\end{array}$ & $\begin{array}{l}9.40 \times 10^{-} \\
03\end{array}$ & -6.67 & $\begin{array}{l}2.48 \times 10^{-} \\
11\end{array}$ \\
\hline & & Quadratic & $\begin{array}{l}5.60 \times 10^{-} \\
04\end{array}$ & $\begin{array}{l}1.26 \times 10^{-} \\
04\end{array}$ & 4.435 & $\begin{array}{l}9.22 \times 10^{-} \\
06\end{array}$ \\
\hline & & Cubic & $\begin{array}{l}-1.75 \times 10^{-} \\
06\end{array}$ & $4.81 \times 10^{-07}$ & -3.641 & $\begin{array}{l}2.71 \times 10^{-} \\
04\end{array}$ \\
\hline & 3rd instar & Intercept & 2.73 & $\begin{array}{l}2.31 \times 10^{-} \\
01\end{array}$ & 11.79 & $<2 \times 10^{-16}$ \\
\hline & & Linear & $\begin{array}{l}-1.04 \times 10^{-} \\
01\end{array}$ & $\begin{array}{l}1.00 \times 10^{-} \\
02\end{array}$ & -10.38 & $<2 \times 10^{-16}$ \\
\hline & & Quadratic & $\begin{array}{l}9.53 \times 10^{-} \\
04\end{array}$ & $\begin{array}{l}1.21 \times 10^{-} \\
04\end{array}$ & 7.859 & $\begin{array}{l}3.86 \times 10^{-} \\
15\end{array}$ \\
\hline
\end{tabular}




\begin{tabular}{|c|c|c|c|c|c|c|}
\hline Temperatures & $\begin{array}{l}\text { Growth } \\
\text { Stages }\end{array}$ & Parameters & Estimates & S.E. & $\begin{array}{l}Z- \\
\text { Value }\end{array}$ & $\operatorname{Pr}(\mathrm{z})$ \\
\hline & & Cubic & $\begin{array}{l}-2.92 \times 10^{-} \\
06\end{array}$ & $\begin{array}{l}4.32 \times 10^{-} \\
07\end{array}$ & -6.749 & $\begin{array}{l}1.49 \times 10^{-} \\
11\end{array}$ \\
\hline & 4th instar & Intercept & 4.12 & $\begin{array}{l}4.02 \times 10^{-} \\
01\end{array}$ & 10.25 & $<2 \mathrm{e}-16$ \\
\hline & & Linear & $\begin{array}{l}-7.67 \times 10^{-} \\
02\end{array}$ & $\begin{array}{l}1.47 \times 10^{-} \\
02\end{array}$ & -5.21 & $\begin{array}{l}1.89 \times 10^{-} \\
07\end{array}$ \\
\hline & & Quadratic & $\begin{array}{l}5.94 \times 10^{-} \\
04\end{array}$ & $\begin{array}{l}1.58 \times 10^{-} \\
04\end{array}$ & 3.755 & $\begin{array}{l}1.73 \times 10^{-} \\
04\end{array}$ \\
\hline & & Cubic & $\begin{array}{l}-1.71 \times 10^{-} \\
06\end{array}$ & $\begin{array}{l}5.18 \times 10^{-} \\
07\end{array}$ & -3.312 & $\begin{array}{l}9.26 \times 10^{-} \\
04\end{array}$ \\
\hline & Male & Intercept & 4.03 & $\begin{array}{l}3.24 \times 10^{-} \\
01\end{array}$ & 12.41 & $<2 \times 10^{-16}$ \\
\hline & & Linear & $\begin{array}{l}-1.13 \times 10^{-} \\
01\end{array}$ & $\begin{array}{l}1.23 \times 10^{-} \\
02\end{array}$ & -9.22 & $<2 \times 10^{-16}$ \\
\hline & & Quadratic & $\begin{array}{l}9.95 \times 10^{-} \\
04\end{array}$ & $\begin{array}{l}1.36 \times 10^{-} \\
04\end{array}$ & 7.307 & $\begin{array}{l}2.73 \times 10^{-} \\
13\end{array}$ \\
\hline & & Cubic & $\begin{array}{l}-2.94 \times 10^{-} \\
06\end{array}$ & $\begin{array}{l}4.58 \times 10^{-} \\
07\end{array}$ & -6.429 & $\begin{array}{l}1.29 \times 10^{-} \\
10\end{array}$ \\
\hline & Female & Intercept & 4.95 & $\begin{array}{l}4.22 \times 10^{-} \\
01\end{array}$ & 11.73 & $<2 \times 10^{-16}$ \\
\hline & & Linear & $\begin{array}{l}-1.28 \times 10^{-} \\
01\end{array}$ & $\begin{array}{l}1.50 \times 10^{-} \\
02\end{array}$ & -8.52 & $<2 \times 10^{-16}$ \\
\hline & & Quadratic & $\begin{array}{l}1.15 \times 10^{-} \\
03\end{array}$ & $\begin{array}{l}1.59 \times 10^{-} \\
04\end{array}$ & 7.259 & $\begin{array}{l}3.89 \times 10^{-} \\
13\end{array}$ \\
\hline & & Cubic & $\begin{array}{l}-3.48 \times 10^{-} \\
06\end{array}$ & $\begin{array}{l}5.18 \times 10^{-} \\
07\end{array}$ & -6.718 & $\begin{array}{l}1.84 \times 10^{-} \\
11\end{array}$ \\
\hline $30^{\circ} \mathrm{C}$ & 1st instar & Intercept & 1.22 & $\begin{array}{l}1.90 \times 10^{-} \\
01\end{array}$ & 6.46 & $\begin{array}{l}1.04 \times 10^{-} \\
10\end{array}$ \\
\hline & & Linear & $\begin{array}{l}-1.00 \times 10^{-} \\
01\end{array}$ & $\begin{array}{l}1.03 \times 10^{-} \\
02\end{array}$ & -9.75 & $<2 \times 10^{-16}$ \\
\hline
\end{tabular}




\begin{tabular}{|c|c|c|c|c|c|c|}
\hline Temperatures & $\begin{array}{l}\text { Growth } \\
\text { Stages }\end{array}$ & Parameters & Estimates & S.E. & $\begin{array}{l}Z- \\
\text { Value }\end{array}$ & $\operatorname{Pr}(\mathrm{z})$ \\
\hline & & Quadratic & $\begin{array}{l}9.454 \times 10^{-} \\
04\end{array}$ & $\begin{array}{l}1.394 \times 10^{-} \\
04\end{array}$ & 6.781 & $\begin{array}{l}1.19 \times 10^{-} \\
11\end{array}$ \\
\hline & & Cubic & $\begin{array}{l}-2.942 \times 10^{-} \\
06\end{array}$ & $\begin{array}{l}5.334 \times 10^{-} \\
07\end{array}$ & -5.515 & $\begin{array}{l}3.48 \times 10^{-} \\
08\end{array}$ \\
\hline & 2nd instar & Intercept & 1.98 & $\begin{array}{l}2.02 \times 10^{-} \\
01\end{array}$ & 9.82 & $<2 \times 10^{-16}$ \\
\hline & & Linear & $\begin{array}{l}-1.05 \times 10^{-} \\
01\end{array}$ & $\begin{array}{l}9.75 \times 10^{-} \\
03\end{array}$ & -10.77 & $<2 \times 10^{-16}$ \\
\hline & & Quadratic & $\begin{array}{l}9.99 \times 10^{-} \\
04\end{array}$ & $\begin{array}{l}1.24 \times 10^{-} \\
04\end{array}$ & 8.060 & $\begin{array}{l}7.61 \times 10^{-} \\
16\end{array}$ \\
\hline & & Cubic & $\begin{array}{l}-3.12 \times 10^{-} \\
06\end{array}$ & $4.56 \times 10^{-07}$ & -6.833 & $\begin{array}{l}8.29 \times 10^{-} \\
12\end{array}$ \\
\hline & 3rd instar & Intercept & 4.40 & $\begin{array}{l}3.25 \times 10^{-} \\
01\end{array}$ & 13.52 & $<2 \times 10^{-16}$ \\
\hline & & Linear & $\begin{array}{l}-1.46 \times 10^{-} \\
01\end{array}$ & $\begin{array}{l}1.26 \times 10^{-} \\
02\end{array}$ & -11.58 & $<2 \times 10^{-16}$ \\
\hline & & Quadratic & $\begin{array}{l}1.34 \times 10^{-} \\
03\end{array}$ & $\begin{array}{l}1.41 \times 10^{-} \\
04\end{array}$ & 9.465 & $<2 \times 10^{-16}$ \\
\hline & & Cubic & $\begin{array}{l}-4.06 \times 10^{-} \\
06\end{array}$ & $\begin{array}{l}4.82 \times 10^{-} \\
07\end{array}$ & -8.420 & $<2 \times 10^{-16}$ \\
\hline & 4th instar & Intercept & 4.97 & $\begin{array}{l}7.80 \times 10^{-} \\
01\end{array}$ & 6.37 & $\begin{array}{l}1.87 \times 10^{-} \\
10\end{array}$ \\
\hline & & Linear & $\begin{array}{l}-3.00 \times 10^{-} \\
02\end{array}$ & $\begin{array}{l}2.86 \times 10^{-} \\
02\end{array}$ & -1.04 & 0.29 \\
\hline & & Quadratic & $6.043 e-05$ & $3.021 \mathrm{e}-04$ & 0.200 & 0.841 \\
\hline & & Cubic & $-2.243 e-07$ & $9.595 \mathrm{e}-07$ & -0.234 & 0.815 \\
\hline & Male & Intercept & 7.65 & $\begin{array}{l}6.89 \times 10^{-} \\
01\end{array}$ & 11.09 & $<2 \times 10^{-16}$ \\
\hline & & Linear & $\begin{array}{l}-2.10 \times 10^{-} \\
01\end{array}$ & $\begin{array}{l}2.25 \times 10^{-} \\
02\end{array}$ & -9.32 & $<2 \times 10^{-16}$ \\
\hline
\end{tabular}




\begin{tabular}{|c|c|c|c|c|c|c|}
\hline \multirow[t]{7}{*}{ Temperatures } & $\begin{array}{l}\text { Growth } \\
\text { Stages }\end{array}$ & Parameters & Estimates & S.E. & $\begin{array}{l}Z- \\
\text { Value }\end{array}$ & $\operatorname{Pr}(\mathrm{z})$ \\
\hline & & Quadratic & $\begin{array}{l}1.87 \times 10^{-} \\
03\end{array}$ & $\begin{array}{l}2.22 \times 10^{-} \\
04\end{array}$ & 8.433 & $<2 \times 10^{-16}$ \\
\hline & & Cubic & $\begin{array}{l}-5.47 \times 10^{-} \\
06\end{array}$ & $\begin{array}{l}6.86 \times 10^{-} \\
07\end{array}$ & -7.986 & $\begin{array}{l}1.4 \times 10^{-} \\
15\end{array}$ \\
\hline & Female & Intercept & 4.96 & $\begin{array}{l}7.32 \times 10^{-} \\
01\end{array}$ & 6.77 & $\frac{1.25 \times 10^{-}}{11}$ \\
\hline & & Linear & $\begin{array}{l}-3.59 \times 10^{-} \\
02\end{array}$ & $\begin{array}{l}2.60 \times 10^{-} \\
02\end{array}$ & -1.38 & 0.16 \\
\hline & & Quadratic & $\begin{array}{l}2.75 \times 10^{-} \\
05\end{array}$ & $\begin{array}{l}2.69 \times 10^{-} \\
04\end{array}$ & 0.102 & 0.919 \\
\hline & & Cubic & $\begin{array}{l}1.38 \times 10^{-} \\
07\end{array}$ & $\begin{array}{l}8.49 \times 10^{-} \\
07\end{array}$ & 0.163 & 0.871 \\
\hline \multirow[t]{9}{*}{$35^{\circ} \mathrm{C}$} & 1 st instar & Intercept & 1.42 & $\begin{array}{l}1.89 \times 10^{-} \\
01\end{array}$ & 7.50 & $\begin{array}{l}6.00 \times 10^{-} \\
14\end{array}$ \\
\hline & & Linear & $\begin{array}{l}-9.36 \times 10^{-} \\
02\end{array}$ & $\begin{array}{l}9.71 \times 10^{-} \\
03\end{array}$ & -9.63 & $<2 \times 10^{-16}$ \\
\hline & & Quadratic & $\begin{array}{l}8.732 \times 10^{-} \\
04\end{array}$ & $\begin{array}{l}1.280 \times 10^{-} \\
04\end{array}$ & 6.823 & $\begin{array}{l}8.92 \times 10^{-} \\
12\end{array}$ \\
\hline & & Cubic & $\begin{array}{l}-2.731 \times 10^{-} \\
06\end{array}$ & $\begin{array}{l}4.828 \times 10^{-} \\
07\end{array}$ & -5.658 & $\begin{array}{l}1.54 \times 10^{-} \\
08\end{array}$ \\
\hline & 2nd instar & Intercept & 2.57 & $\begin{array}{l}2.22 \times 10^{-} \\
01\end{array}$ & 11.57 & $<2 \times 10^{-16}$ \\
\hline & & Linear & $\begin{array}{l}-1.15 \times 10^{-} \\
01\end{array}$ & $\begin{array}{l}1.01 \times 10^{-} \\
02\end{array}$ & -11.37 & $<2 \times 10^{-16}$ \\
\hline & & Quadratic & $\begin{array}{l}1.089 \times 10^{-} \\
03\end{array}$ & $\begin{array}{l}1.25 \times 10^{-} \\
04\end{array}$ & 8.706 & $<2 \times 10^{-16}$ \\
\hline & & Cubic & $\begin{array}{l}-3.37 \times 10^{-} \\
06\end{array}$ & $\begin{array}{l}4.52 \times 10^{-} \\
07\end{array}$ & -7.458 & $\begin{array}{l}8.77 \times 10^{-} \\
14\end{array}$ \\
\hline & 3rd instar & Intercept & 4.00 & $\begin{array}{l}3.12 \times 10^{-} \\
01\end{array}$ & 12.81 & $<2 \times 10^{-16}$ \\
\hline
\end{tabular}




\begin{tabular}{|c|c|c|c|c|c|c|}
\hline Temperatures & $\begin{array}{l}\text { Growth } \\
\text { Stages }\end{array}$ & Parameters & Estimates & S.E. & $\begin{array}{l}Z \text { - } \\
\text { Value }\end{array}$ & $\operatorname{Pr}(\mathrm{z})$ \\
\hline & & Linear & $\begin{array}{l}-1.21 \times 10^{-} \\
01\end{array}$ & $\begin{array}{l}1.21 \times 10^{-} \\
02\end{array}$ & -10.03 & $<2 \times 10^{-16}$ \\
\hline & & Quadratic & $\begin{array}{l}1.05 \times 10^{-} \\
03\end{array}$ & $\begin{array}{l}1.36 \times 10^{-} \\
04\end{array}$ & 7.714 & $\begin{array}{l}1.22 \times 10^{-} \\
14\end{array}$ \\
\hline & & Cubic & $\begin{array}{l}-3.11 \times 10^{-} \\
06\end{array}$ & $\begin{array}{l}4.66 \times 10^{-} \\
07\end{array}$ & -6.672 & $\begin{array}{l}2.52 \times 10^{-} \\
11\end{array}$ \\
\hline & 4th instar & Intercept & 6.90 & 1.07 & 6.44 & $\begin{array}{l}1.13 \times 10^{-} \\
10\end{array}$ \\
\hline & & Linear & $\begin{array}{l}-1.22 \times 10^{-} \\
01\end{array}$ & $\begin{array}{l}3.59 \times 10^{-} \\
02\end{array}$ & -3.40 & 0.0006 \\
\hline & & Quadratic & $\begin{array}{l}1.14 \times 10^{-} \\
03\end{array}$ & $\begin{array}{l}3.59 \times 10^{-} \\
04\end{array}$ & 3.173 & $\begin{array}{l}1.50 \times 10^{-} \\
04\end{array}$ \\
\hline & & Cubic & $\begin{array}{l}-3.75 \times 10^{-} \\
06\end{array}$ & $\begin{array}{l}1.11 \times 10^{-} \\
06\end{array}$ & -3.385 & $\begin{array}{l}7.13 \times 10^{-} \\
04\end{array}$ \\
\hline & Male & Intercept & 9.20 & 1.15 & 7.94 & $\begin{array}{l}1.94 \times 10^{-} \\
15\end{array}$ \\
\hline & & Linear & $\begin{array}{l}-2.25 \times 10^{-} \\
01\end{array}$ & $\begin{array}{l}3.61 \times 10^{-} \\
02\end{array}$ & -6.25 & $\begin{array}{l}4.08 \times 10^{-} \\
10\end{array}$ \\
\hline & & Quadratic & $\begin{array}{l}1.93 \times 10^{-} \\
03\end{array}$ & $\begin{array}{l}3.41 \times 10^{-} \\
04\end{array}$ & 5.660 & $\begin{array}{l}1.51 \times 10^{-} \\
08\end{array}$ \\
\hline & & Cubic & $\begin{array}{l}-5.50 \times 10^{-} \\
06\end{array}$ & $\begin{array}{l}1.01 \times 10^{-} \\
06\end{array}$ & -5.437 & $\begin{array}{l}5.43 \times 10^{-} \\
08\end{array}$ \\
\hline & Female & Intercept & 5.13 & $\begin{array}{l}8.52 \times 10^{-} \\
01\end{array}$ & 6.02 & $\begin{array}{l}1.72 \times 10^{-} \\
09\end{array}$ \\
\hline & & Linear & $\begin{array}{l}-2.91 \times 10^{-} \\
02\end{array}$ & $\begin{array}{l}3.14 \times 10^{-} \\
02\end{array}$ & -0.92 & 0.35 \\
\hline & & Quadratic & $\begin{array}{l}6.93 \times 10^{-} \\
05\end{array}$ & $\begin{array}{l}3.31 \times 10^{-} \\
04\end{array}$ & 0.209 & 0.834 \\
\hline & & Cubic & $\begin{array}{l}-3.27 \times 10^{-} \\
07\end{array}$ & $\begin{array}{l}1.05 \times 10^{-} \\
06\end{array}$ & -0.311 & 0.756 \\
\hline
\end{tabular}


Estimates of functional response parameters, determined through Rogers random predator model, revealed that the $H$. axyridis exhibited the highest attack rate (Fig. 7a), the shortest handling time (Fig. 7b), and the maximum predation (Fig. 7c) at higher temperatures, and typically at later growth stages. The outcomes of all three parameters were generally low at low thermal conditions $\left(15\right.$ and $\left.20^{\circ} \mathrm{C}\right)$ but started to improve with warming, with the best results at 30 and $35^{\circ} \mathrm{C}$. The outcomes of all three parameters were much low for 1st, 2nd, and 3rd instar when compared with the 4th instar and adult $H$. axyridis. Female $H$. axyridis performed much better than male $H$. axyridis, especially in terms of handling time and maximum predation rate (Fig. 7). The attack rate of 4 th instar (i.e., $0.234 \pm 0.014 \mathrm{~h}^{-1}$ ) was greatest at $30^{\circ} \mathrm{C}$, whereas of adult male (i.e., $0.180 \pm 0.01 \mathrm{~h}^{-1}$ ) and female (i.e., $0.247 \pm 0.015 \mathrm{~h}^{-1}$ ) was at $35^{\circ} \mathrm{C}$. The handling time of 4 th instar (i.e., $0.132 \pm 0.005 \mathrm{~h}$ ) and adult female (i.e., $0.156 \pm 0.004 \mathrm{~h}$ ) was shortest at $35^{\circ} \mathrm{C}$. The maximum predation rate for 4 th instar (i.e., $181.28 \pm 14.54$ ), adult male (i.e., $91.90 \pm$ 21.48 ) and female (i.e., $153.85 \pm 4.06$ ) was obtained at $35^{\circ} \mathrm{C}$.

\section{Discussion}

Exploring the temperature influence on predator-prey interactions ${ }^{16}$ can be highly insightful towards conservative or augmentative biocontrol programs ${ }^{54}$. As insects are ectotherms, their development and biology are responsive towards temperature ${ }^{55}$, so are their behavioral trophic interactions ${ }^{16,56}$. Theories concerning temperature change and its influence on ecosystem functions, including foraging interactions ${ }^{57}$, are gaining increasing prominence since the past decade ${ }^{30,31}$. The current research is the first report exploring functional response of $H$. axyridis to A. pisum at different growth stages and temperatures. The temperature ranges we have tested are existent across a range of temperate or subtropical regions. We determined increasing host aphid mortality with warming, meaning warming can lead towards prey depletion. A low-temperature threshold (i.e., $20^{\circ} \mathrm{C}$ ) is reported the best for aphid growth and development, whereas, higher temperatures $>30^{\circ} \mathrm{C}$ become unfavorable for aphid fertility and development, and discourage its population buildup ${ }^{93}$. We showed a Type II functional response by all stages of $\mathrm{H}$. axyridis, within the tested host aphid density ranges and thermal conditions, with maximum predation by the 4th instar and female $\mathrm{H}$. axyridis between 25 and $35^{\circ} \mathrm{C}$. Warming is shown to accelerate insect metamorphosis. Accelerated growth/development enhances metabolic rate and energy gain requirements ${ }^{50,58}$, which the predator meets by consuming large meals ${ }^{59}$, explaining our results of heightened predation under warming. Heightened predation under warming (between 14 and $35^{\circ} \mathrm{C}$ ), is already known for $H$. axyridis preying on aphids $C$. juglandicola or $P$. juglandis ${ }^{46}$, or eggs of Spodoptera litura F. (Lepidoptera: Noctuidae) ${ }^{50}$, and for other coccinellids, Adalia bipunctata L., Hippodamia convergens Guérin-Méneville and Coccinella septempunctata L., preying on aphid Myzus persicae $(\text { Sulzer })^{26,60}$.

Functional response models are employed to quantifying the consumption relationship between a predator and its resource ${ }^{12}$, and a typical model illustrating these relations is the Type II, explaining a curvilinear increase in predation with respect to increasing prey density, changing to asymptotic at high 
prey densities, and thenceforth hanging around constant by reason of satiation ${ }^{12}$. A Type II response is often a characteristic of those predators that provide efficient control at smaller resource ${ }^{61}$, though often associated with unstable predator-prey population dynamics ${ }^{17,62,63}$, owing to the decreasing risk of predation with resource abundance. This means a negative density-dependent mortality, which can unstable population dynamics ${ }^{64}$. Many sources of variations are shown to have regulatory roles towards functional response, including prey/predator biology, switching, preference, host distribution, patch allocation time of predator, and intra/interspecific competitions ${ }^{65-67}$.

In our findings, despite its positive role towards consumption, no change in $\mathrm{H}$. axyridis functional response type (i.e., Type II) was observed towards thermal changes. A changing functional response type with thermal changes has been reported for many predators ${ }^{29}$, but rarely for coccinellids. Harmonia axyridis when preying on immature $S$. litura ${ }^{50}$, or when preying on $C$. juglandicola or $P$. juglandis with respect to the temperature ${ }^{46}$, Rhopalosiphum padi L. and Sitobion avenae F. to the fertilizer treatments ${ }^{68}$, Lipaphis erysimi K. (Hemiptera: Aphididae), Cacopsylla chinensis (Yang \& Li) (Hemiptera: Psyllidae) and Danaus plexippus L. (Lepidoptera: Nymphlidae) to prey identity $51,69,70$, A. gossypii, Myzus persicae S., M. nicotianae S., Aphis glycines Matsumura (Hemiptera: Aphididae) and Diaphorina citri (Hemiptera: Psyllidae) to growth stage ${ }^{71-73}$ have showed frequently a Type II functional response ${ }^{65}$. However, the change of functional response type with respect to prey distribution ${ }^{74}$ or prey quality (either pesticide treated or untreated) have been shown for Harmonia axyridis ${ }^{48,49}$, suggesting complex nature of predatorprey interactions, and, emphasizing the need for further assessments of current findings with regard to general factors like pesticides ${ }^{75}$. Insecticides from synthetics and biopesticides groups are commonly applied worldwide ${ }^{76-78}$ and shown to have profound effects, both positive or negative, on behavioral or physiological responses of predators ${ }^{45,79}$.

The attack rate and handling time are important parameters, describing functional response magnitude. The attack rate (also called the space clearance rate or attack efficiency) describes the ability a predator possesses to catch its prey in a given time frame, and handling time describes the time lost from searching per host consumed ${ }^{80}$. A high attack rate means that the predator is adept at quickly removing hosts from the volumes or areas it is searching, and low handling time means how quickly a predator traps, hunts, and digests prey ${ }^{80}$. In our findings, the parameter estimates showed greater variations across predator growth stages, with frequently better estimates at later growth stages and higher temperatures. Maximum daily predation rates were temperature-dependent, especially their increase, and the handling time of $\mathrm{H}$. axyridis presented an exponential decrease for all growth stages at the lowest thermal conditions, meaning predators will spend less investment on foraging, convert that to resting, for example, and decrease predation event ${ }^{81,82}$. Conversely, the greater number of prey consumed owing to the warming implies a decrease in handling time with a resultant increase in time to approach other prey at alleviated temperature ${ }^{83}$. The increased metabolic rates under warming were associated with greater energy demands, which should expectedly maximize food intake and foraging activity. Determining these 
reported earlier ${ }^{72,84}$, whereas better by final instar and adult $H$. axyridis (especially female when compared with male) ${ }^{85,86}$ suggesting $H$. axyridis better biocontrol efficiency at later stages, plausibly owing to better searching efficiencies and agilities. The 4th instar requires large meals to attain the required weight for pupation ${ }^{87}$ and adult predators have to prepare for reproduction ${ }^{69}$ and other functions related to egg maturation or fertilization ${ }^{88}$.

Though employed commonly to understand the predator-prey interactions related to food consumption and food web stability ${ }^{89,90}$, the functional response models are usually applied under controlled conditions and do not thoroughly represent the field circumstances, hence fail to count for complex and diverse ecological interactions. For example, small-scale setups (such as Petri dish) may not mimic the actual field conditions ${ }^{91}$, and a predator with abundant prey resource, as under controlled conditions, will never going to experience the challenges of emigration or cannibalism ${ }^{92}$, which could occur under poor resource availability. Despite these limitations, the functional response models allow us to understand complex foraging interactions and forecast ecosystem stability. We determined the strong efficiency of $H$. axyridis towards $A$. pisum control, but the predation activity depended upon the growth stage and thermal conditions imposed. The 4th instar and female $H$. axyridis emerged as the best performing biocontrol candidates, with best efficiencies under warming conditions. Another striking effect of warming was determined on prey mortality that increased with warming. This implies that warming may lead towards aphid depletion and consequently trigger intraguild predation or other antagonistic interactions among predator populations ${ }^{94}$. This allows us to suggest that $H$. axyridis can be used at low temperatures (between 20 and $25^{\circ} \mathrm{C}$ ), but the risk of intraguild predation can be expected at higher temperatures. Provision with alternative prey resources could be a feasible way of supporting this generalist predator without changing functional response type; however, further research is needed to synthesize careful conclusions. Thus, we recommend further studies to evaluate current findings with the inclusion of the other contributable factors, such as intraguild predation, alternative prey resource, mutual interference, pesticides, etc., so that the actual predation behaviour of $H$. axyridis to A. pisum may be approached and an efficient biological control may be developed and implemented.

\section{Methods}

Cucumber (Cucumis sativa L., cv. Negin; Cucurbitales: Cucurbitaceae) and broad bean (Vicia faba L.; Fabales: Fabaceae) seedlings were grown from seeds purchased from Caoxian County, Shandong, China. The seeds were sown in $30 \times 25 \mathrm{~cm}$ diameter regular pots filled with (3:1) soil: manure. The seedlings were maintained under greenhouse conditions of $12-26^{\circ} \mathrm{C}, 45-55 \% \mathrm{RH}$, and 16:8 $\mathrm{h}$ (Light: Dark) photoperiod, and subsequently used for rearing and conducting functional response assays. The plant materials used were obtained with prior permission, and the present study is in compliance with relevant guidelines and legislation.

For establishing $A$. pisum culture, the initial populations of aphid collected from unsprayed alfalfa fields woro cuhconuontly hrowaht to tho lahnratnry and reared on broad bean plants inside net cages $(20 \times 10 \times$ Loading [MathJax]/jax/output/CommonHTML/jax.js 
$30 \mathrm{~cm}$ height). The stock culture of $H$. axyridis was developed from a pre-established laboratory colony, already available in the same laboratory. The predator was reared on A. pisum infested bean plants (7-8 leaves) inside net cages $(60 \times 42 \times 30 \mathrm{~cm}$ height) for three consecutive generations at laboratory conditions of $24 \pm 1^{\circ} \mathrm{C}, 65 \pm 5 \% \mathrm{RH}$ and $16: 8 \mathrm{~h}$ (Light: Dark) photoperiod. Bean plants were checked daily for predator eggs. Egg batches when found were carefully removed, placed on tissue paper in Petri dishes $(9 \mathrm{~cm})$, and transferred to a computer-operated growth chamber, maintained at settings of $25 \pm 1^{\circ} \mathrm{C}, 65 \pm 5$ $\% \mathrm{RH}$ and 16:8 h (Light: Dark) photoperiod. The post-emergence larvae were separated and reared in Petri dishes containing aphid as their diet, refreshed daily. The whole culture was maintained at the Department of Plant Protection, Huazhong Agricultural University, China.

The experimental arena consisted of clear Petri dishes ( $9 \mathrm{~cm}$ diameter), with a micromesh screen over the top for ventilation and bottom covered with clean cucumber leaf disk. The desiccation of cucumber leaf disc was prevented by adding $1 \%$ agar solution ${ }^{95}$. The assays were performed with $H$. axyridis larvae (i.e., 1st instar, 2nd instar, 3rd instar, 4th instar) and adults (male, female) at constant temperatures (i.e., 15, $20,25,30,35^{\circ} \mathrm{C}$ ). The homogeneity of predator age was maintained within each tested growth stage. The first instar larvae were separated one by one shortly after hatching to avoid sibling cannibalism. Hatchlings were reared in Petri dishes ( $9 \mathrm{~cm}$ diameter) until maturity on 4th instar nymphs (100-150 aphids/day). Female $H$. axyridis included mated individuals ${ }^{72}$. First instar larvae were starved for about $6 \mathrm{~h}$, whereas subsequent instars/stages were starved for $24 \mathrm{~h}$ to standardize hunger level, according to Islam, et al. ${ }^{50}$. The moist cotton roll offered humidity to all predators during starvation. The use of 4 th instar aphid was ensured throughout the experiments as a way to prevent predator preference switch according to prey size $e^{96}$. Using a fine camel hairbrush, the aphids at different densities (i.e., 2, 4, 8, 16, 32, 64,128 , and 160 aphids) were transferred in Petri dishes, allowed for 30 minutes to uniformly spread and settle over the substrate, and thereafter were transferred to a computerized growth chamber at various fixed temperatures (i.e., 15, 20, 25, 30, 35 ${ }^{\circ} \mathrm{C}$ ), $70 \pm 5 \% \mathrm{RH}$ and 16:8h (Light: Dark) photoperiod. The whole experiment was replicated 10 times for each prey density, growth stage, and temperature. The numbers of aphid consumed were recorded every 24th $\mathrm{h}$. Control replicates were kept free from $\mathrm{H}$. axyridis to account for natural mortality and correct $A$. pisum consumption by the predator as a function of natural mortality. Predation mortality data were corrected for control mortality by applying Abbott's correction ${ }^{97}$.

The control mortality data were analyzed between temperatures, aphid densities, and their interaction, by using Univariate Analysis of Variance (ANOVA) in SPSS (version 21), fitting the above three variables as fixed factors against the dependent variable (i.e., host mortality). Significant $(P<0.05)$ effects were further compared by using Tukey's Honestly Significant Difference (HSD) multiple comparisons Test. Prior to analysis, the mortality data were tested for normality and homogeneity of error variance (i.e., homoscedasticity) by using Shapiro-Wilk and Levene tests, and $Y=\sqrt{ } x+1$ transformed to improve compliance with these assumptions. All means and standard errors in text and figures are calculated with untransformed data. 
Aphid consumption by $H$. axyridis for temperature, growth stage, density, and their two-way and three-way interactions were analyzed by using Generalized Linear Models (GLM) in SPSS (version 21). KolmogorovSmirnov test confirmed non-normal distributions of data $(P>0.05)$, and due to over-dispersion, the data were fitted with negative binomial distribution and a log link function, and factors and interaction effects were analyzed by using the Wald Chi-Square test for a confidence level $(\mathrm{Cl})$ of $95 \%$. If needed, the multiple follow up tests were run to analyze the temperature and growth stage effects, separately, at each aphid density, and the significance for each test was adjusted by following Bonferroni correction to avoid Type 1 error.

Analysis of the functional response was done in two different phases ${ }^{17}$ : first phase involved the determination of type and estimation of the parameters of the functional response curve. It is compulsory to find the type of functional response for calculating the functional response parameters using a proper model. The type was determined by applying logistic regression of the proportion of prey eaten as a function of initial prey density offered. A polynomial logistic regression equation assuming a binomial distribution of data to define the type of functional response ${ }^{17}$ (Eq. 1) was fitted as under:

$$
\frac{N_{a}}{N_{0}}=\frac{\exp \left(P_{o}+P_{1} N_{o}+P_{2} N_{o}^{2}+P_{3} N_{o}^{\mathrm{a}}\right)}{1+\exp \left(P_{o}+P_{1} N_{o}+P_{2} N_{o}^{2}+P_{3} N_{o}^{\mathrm{g}}\right)}
$$

Where $N_{a}$ and $N_{o}$ indicate the number of prey consumed and the initial prey density offered, respectively, and $\frac{N a}{N o}$ is the proportion of prey consumed. The $P_{0}, P_{1}, P_{2}$, and $P_{3}$ are the regression parameters representing intercept or constant, linear, quadratic, and cubic coefficients, respectively. The coefficients were calculated by using the maximum likelihood method. The values of the linear and quadratic coefficients indicate the nature of functional response either it is Type II or Type III. When the value of a linear parameter is negative, the functional response is Type II, and if it is positive with a negative quadratic coefficient, then response is of Type III. The Type II response shows that the proportion of prey consumption decreases as the prey density increases, and a Type III response represents that the proportion of prey consumed increases until an inflection point and then decreases ${ }^{17}$. Once the functional response type was determined, the second phase started where functional response parameters were determined. For which, data were fitted to Rogers' type II random predator equation, with the help of nonlinear least square regression, and determined and analyzed the parameters of functional response. As the prey was not changed or replaced during the entire experiment, the random predator equation was determined to be more appropriate for such a dataset ${ }^{98}$. The attack rate $(a)$ and handling time $(T h)$ were calculated by using the random predator model as under: (Eq. 2).

$$
N_{a}=N_{o}\left[1-\exp \left(a\left(T_{h} N_{a}-T\right)\right)\right]
$$

Where, $a$ is the attack rate, $T_{h}$ is the handling time, $T$ is time available for predator during the experiment. Here, " $g / m$ " function was used to fit the logistic regression, and the parameters (attack rate $a$ and handlina time $T_{h}$ of functional response were estimated by using FRAIR (Functional Response Analysis in Loading [MathJax]/jax/output/CommonHTML/jax.js 
$\mathrm{R}$, version 4.0.0 $)^{99}$ in the $\mathrm{R}$ statistical environment ${ }^{100}$. The maximum predation rate is the ratio between $T / T h^{101}$ and estimates the maximum amount of prey that a predator can consume in a given time frame.

\section{Declarations}

Funding: This research was funded by the National Key R and D Program of China (2017YFD0201000), The National Natural Science Foundation of China, Grant No. 31872023, and the Key Research Program of Hubei Tobacco Company (027Y2018-008).

\section{Acknowledgments:}

We greatly acknowledge the help and support from Dr. John Paul DeLong (Associate Professor, DirectorCedar Point Biological Station, University of Nebraska-Lincoln, USA) for his valuable comments on the initial draft. We offer our thanks to colleagues and friends who helped during this research, especially M. Abas Shah, for his help regarding processing of data in R. We extend our special thanks to Dr. Afifa Naeem (Entomological Research Institute, Ayub Agricultural Research Institute, Faisalabad, Punjab, Pakistan) for helping with proof reading the manuscript.

\section{Author Contributions}

$\mathrm{XZ}$ conceived the idea and supervised the laboratory trials. YI and FMS designed the protocol of laboratory experiments. YI performed the experiments, analyzed the data in R software, and wrote the initial draft. FMS and XR analyzed the data in SPSS software, and wrote the final draft. MR, XR, MY, LX and $X Z$ reviewed the manuscripts. All authors discussed and approved the publication of the manuscript.

\section{Competing Interests}

The authors declare there are no competing interests.

\section{References}

1. van Lenteren, J. C., Bolckmans, K., Köhl, J., Ravensberg, W. J. \& Urbaneja, A. Biological control using invertebrates and microorganisms: plenty of new opportunities. BioControl. 63, 39-59 (2018).

2. Cock, M. J. et al. Trends in the classical biological control of insect pests by insects: an update of the BIOCAT database. BioControl. 61, 349-363 (2016).

3. Zang, L. S., Wang, S., Zhang, F. \& Desneux, N. Biological control with Trichogramma in China: history, present status and perspectives. Annual Review of Entomology. https://doi.org/10.1146/annurevento-060120-091620 (2020).

4. Gabarra, R., Alomar, Ã., Castañé, C., Goula, M. \& Albajes, R. Movement of greenhouse whitefly and its predators between in-and outside of Mediterranean greenhouses. Agriculture, ecosystems \& environment. 102, 341-348 (2004). 
5. Sindhu, S. S., Sehrawat, A., Sharma, R. \& Khandelwal, A. in Advances in Soil Microbiology: Recent Trends and Future Prospects 189-218(Springer, 2017).

6. Bale, J., Van Lenteren, J. \& Bigler, F. Biological control and sustainable food production. Philosophical Transactions of the Royal Society B: Biological Sciences. 363, 761-776 (2008).

7. Conti, E. et al. Biological control of invasive stink bugs: review of global state and future prospects. Entomologia Experimentalis et Applicata. 169, 28-51 (2021).

8. Conti, E. \& Wajnberg, E. \& Beukeboom, L. W. Entomophagous insects-an introduction.Entomologia Experimentalis et Applicata(2021).

9. Gibert, J. P. Temperature directly and indirectly influences food web structure. Scientific reports. 9, 18 (2019).

10. Wootton, J. T. \& Emmerson, M. Measurement of interaction strength in nature. Annu. Rev. Ecol. Evol. Syst. 36, 419-444 (2005).

11. Novak, M. \& Wootton, J. T. Using experimental indices to quantify the strength of species interactions. Oikos. 119, 1057-1063 (2010).

12. Holling, C. S. Some characteristics of simple types of predation and parasitism. Canadian entomologist. 91, 385-398 (1959).

13. Fathipour, Y., Maleknia, B., Bagheri, A., Soufbaf, M. \& Reddy, G. V. Functional and numerical responses, mutual interference, and resource switching of Amblyseius swirskii on two-spotted spider mite. Biol. Control. 146, 104266 (2020).

14. Van Lenteren, J. C. et al. Pest kill rate as aggregate evaluation criterion to rank biological control agents: A case study with Neotropical predators of Tuta absoluta on tomato. Bulletin of entomological research. 109, 812-820 (2019).

15. Xia, P. L., Yu, X. L., Li, Z. T. \& Feng, Y. The impacts of Harmonia axyridis cues on foraging behavior of Aphidius gifuensis to Myzus persicae. Journal of Asia-Pacific Entomology. 24, 278-284 (2021).

16. Uiterwaal, S. F. \& DeLong, J. P. Functional responses are maximized at intermediate temperatures. Ecology. 101, e02975 (2020).

17. Juliano, S. A. Nonlinear curve fitting: predation and functional response curves. Design and analysis of ecological experiments. 2, 178-196 (2001).

18. Jeschke, J. M. \& Tollrian, R. Effects of predator confusion on functional responses. Oikos. 111, 547555 (2005).

19. Pervez, A. Functional responses of coccinellid predators: an illustration of a logistic approach. Journal of Insect Science. 5, 5 (2005).

20. Fritz, H., Durant, D. \& Guillemain, M. Shape and sources of variations of the functional response of wildfowl: an experiment with mallards, Anas platyrhynchos. Oikos. 93, 488-496 (2001).

21. Uiterwaal, S. F. \& DeLong, J. P. Multiple factors, including arena size, shape the functional responses of ladybird beetles. Journal of Applied Ecology. 55, 2429-2438 (2018). 
22. Diamond, S. E. Contemporary climate-driven range shifts: Putting evolution back on the table. Funct. Ecol. 32, 1652-1665 (2018).

23. Andrew, N. R. et al. Assessing insect responses to climate change: What are we testing for? Where should we be heading? PeerJ 1, e11(2013).

24. Parajulee, M., Shrestha, R., Leser, J., Wester, D. \& Blanco, C. Evaluation of the functional response of selected arthropod predators on bollworm eggs in the laboratory and effect of temperature on their predation efficiency. Environmental entomology. 35, 379-386 (2006).

25. Forster, J. \& Hirst, A. G. The temperature-size rule emerges from ontogenetic differences between growth and development rates. Funct. Ecol. 26, 483-492 (2012).

26. Jalali, M. A., Tirry, L. \& De Clercq, P. Effect of temperature on the functional response of Adalia bipunctata to Myzus persicae. BioControl. 55, 261-269 (2010).

27. Moezipour, M., Kafil, M. \& Allahyari, H. Functional response of Trichogramma brassicae at different temperatures and relative humidities. Bulletin of Insectology. 61, 245-250 (2008).

28. Clercq, D. Functional response of the predators Podisus maculiventris (Say) and Podisus nigrispinus (Dallas)(Het., Pentatomidae) to the beet armyworm, Spodoptera exigua (Hübner)(Lep., Noctuidae): effect of temperature. Journal of Applied Entomology. 125, 131-134 (2001).

29. da Silva Nunes, G. et al. Temperature-dependent functional response of Euborellia annulipes (Dermaptera: Anisolabididae) preying on Plutella xylostella (Lepidoptera: Plutellidae) larvae. Journal of Thermal Biology. 93, 102686 (2020).

30. Murrell, E. G. \& Barton, B. T. Warming alters prey density and biological control in conventional and organic agricultural systems. Integrative and comparative biology. 57, 1-13 (2017).

31. Damien, M. \& Tougeron, K. Prey-predator phenological mismatch under climate change. Current opinion in insect science. 35, 60-68 (2019).

32. Thomas, C. A list of the species of the tribe Aphidini, family Aphidae, found in the United States, which have been heretofore named, with descriptions of some new species. Illinois Natural History Survey Bulletin. 1, 3-16 (1878).

33. Elbakidze, L., Lu, L. \& Eigenbrode, S. Evaluating vector-virus-yield interactions for peas and lentils under climatic variability: a limited dependent variable analysis. Journal of Agricultural and Resource Economics,504-520(2011).

34. Aznar-Fernández, T., Cimmino, A., Masi, M., Rubiales, D. \& Evidente, A. Antifeedant activity of longchain alcohols, and fungal and plant metabolites against pea aphid (Acyrthosiphon pisum) as potential biocontrol strategy. Natural product research. 33, 2471-2479 (2019).

35. Wang, D. et al. Identification and virulence characterization of entomopathogenic fungus Lecanicillium attenuatum against the pea aphid Acyrthosiphon pisum (Hemiptera: Aphididae). Applied Entomology and Zoology. 52, 511-518 (2017).

36. Paudel, S., Bechinski, E. J., Stokes, B. S., Pappu, H. R. \& Eigenbrode, S. D. Deriving economic models for pea aphid (Hemiptera: Aphididae) as a direct-pest and a virus-vector on commercial lentils.

Loading [MathJax]/jax/output/CommonHTML/jax.js 2225-2232 (2018).

Page 20/30 
37. Rashed, A. et al. Vector-borne viruses of pulse crops, with a particular emphasis on North American cropping system. Annals of the Entomological Society of America. 111, 205-227 (2018).

38. Van Emden, H. \& Harrington, R. (Oxford, 2007).

39. Anuj, B. Efficacy and economics of some insecticides and a neem formulation on incidence of pea aphid (Acyrthosiphum pisum) on pea, Pisum sativum. Annals of Plant Protection Sciences. 4, 131133 (1996).

40. Slusher, E. K., Cottrell, T. \& Acebes-Doria, A. L. Effects of Aphicides on Pecan Aphids and Their Parasitoids in Pecan Orchards. Insects. 12, 241 (2021).

41. Soleimani, S. \& Madadi, H. Seasonal dynamics of: the pea aphid, Acyrthosiphon pisum (Harris), its natural enemies the seven spotted lady beetle Coccinella septempunctata Linnaeus and variegated lady beetle Hippodamia variegata Goeze, and their parasitoid Dinocampus coccinellae (Schrank). Journal of Plant Protection Research55 (2015).

42. Brown, P. M. et al. The global spread of Harmonia axyridis (Coleoptera: Coccinellidae): distribution, dispersal and routes of invasion. BioControl. 56, 623-641 (2011).

43. Jianhua, D., Zhongxia, T., Qiongli, S., Xingfu, W. \& Jiang, L. Functional Responses and Density Interference Effect in Harmonis axyridis Pallas A Predator to Myzus nicotianae (Blackman). Xi nan Nong ye da xue xue bao = Journal of Southwest Agricultural University. 24, 433-435 (2002).

44. Wu, P. et al. Possible coexistence of Harmonia axyridis Pallas (Coleoptera: Coccinellidae) and Diaeretiella rapae M'Intosh (hymenoptera: Braconidae) in the biological control of Lipaphis erysimi (Homoptera: Aphididae). Journal of Asia-Pacific Entomology. 22, 250-255 (2019).

45. Rasheed, M. A. et al. Lethal and Sublethal Effects of Chlorpyrifos on Biological Traits and Feeding of the Aphidophagous Predator Harmonia axyridis. Insects. 11, 491 (2020).

46. Gao, G., Liu, S., Feng, L., Wang, Y. \& Lu, Z. Effect of temperature on predation by Harmonia axyridis (Pall.)(Coleoptera: Coccinellidae) on the walnut aphids Chromaphis juglandicola Kalt. and Panaphis juglandis (Goeze). Egyptian Journal of Biological Pest Control. 30, 1-6 (2020).

47. Dargazani, B. \& Sahragard, A. Life table and predation rate of Harmonia axyridis spectabilis (col., Coccinellidae), fed on Aphis gossypii (Hem., Aphididae). Archives of Phytopathology and Plant Protection. 53, 282-297 (2020).

48. Qin, D. et al. Treating green pea aphids, Myzus persicae, with azadirachtin affects the predatory ability and protective enzyme activity of harlequin ladybirds, Harmonia axyridis. Ecotoxicology and Environmental Safety. 212, 111984 (2021).

49. Dai, C. et al. Can contamination by major systemic insecticides affect the voracity of the harlequin ladybird? Chemosphere. 256, 126986 (2020).

50. Islam, Y. et al. Temperature-dependent functional response of Harmonia axyridis (Coleoptera: Coccinellidae) on the eggs of Spodoptera litura (Lepidoptera: Noctuidae) in laboratory. Insects. 11, $583(2020)$.

51. Ge, Y. et al. Different predation capacities and mechanisms of Harmonia axyridis (Coleoptera:

Loading [MathJax]/jax/output/CommonHTML/jax.js pear psylla Cacopsylla chinensis (Hemiptera: Psyllidae). PLoS 
ONE. 14, e0215834 (2019).

52. Khan, M. H. \& Yoldaş, Z. Assessment of the functional response parameters of Coccinella septempunctata to varying densities of Acyrthosiphon pisum. Journal of Asia-Pacific Entomology. 21, 1165-1170 (2018).

53. Ünlü, A. G., Terlau, J. F. \& Bucher, R. Predation and avoidance behavior of the pea aphid Acyrthosiphon pisum confronted with native and invasive lady beetles in Europe.Biological Invasions, 1-10(2020).

54. Schulz, A. N., Lucardi, R. D. \& Marsico, T. D. Strengthening the Ties That Bind: An Evaluation of Crossdisciplinary Communication Between Invasion Ecologists and Biological Control Researchers in Entomology.Annals of the Entomological Society of America(2021).

55. Sinclair, B. J., Williams, C. M. \& Terblanche, J. S. Variation in thermal performance among insect populations. Physiological and Biochemical Zoology. 85, 594-606 (2012).

56. Logan, J. D., Wolesensky, W. \& Joern, A. Temperature-dependent phenology and predation in arthropod systems. Ecological modelling. 196, 471-482 (2006).

57. Chen, X., Wong, S. W. \& Stansly, P. A. Functional response of Tamarixia radiata (Hymenoptera: Eulophidae) to densities of its host, Diaphorina citri (Hemiptera: Psylloidea). Annals of the Entomological Society of America. 109, 432-437 (2016).

58. Seyfollahi, F., Esfandiari, M., Mossadegh, M. \& Rasekh, A. Functional response of Hyperaspis polita (Coleoptera, Coccinellidae) to the recently invaded mealybug Phenacoccus solenopsis (Hemiptera, Pseudococcidae). Neotropical entomology. 48, 484-495 (2019).

59. Walker, R., Wilder, S. M. \& González, A. L. Temperature dependency of predation: Increased killing rates and prey mass consumption by predators with warming. Ecology and Evolution(2020).

60. Katsarou, I., Margaritopoulos, J. T., Tsitsipis, J. A., Perdikis, D. C. \& Zarpas, K. D. Effect of temperature on development, growth and feeding of Coccinella septempunctata and Hippodamia convergens reared on the tobacco aphid, Myzus persicae nicotianae. BioControl 50, 565-588(2005).

61. Koehler, H. Predatory mites (Gamasina, Mesostigmata). Agric. Ecosyst. Environ. 74, 395-410 (1999).

62. Nachman, G. A functional response model of a predator population foraging in a patchy habitat. Journal of Animal Ecology. 75, 948-958 (2006).

63. Briggs, C. J. \& Hoopes, M. F. Stabilizing effects in spatial parasitoid-host and predator-prey models: a review. Theoretical population biology. 65, 299-315 (2004).

64. Hassell, M. P. The dynamics of arthopod predator-prey systems(1978).

65. Obrycki, J. J. \& Kring, T. J. Predaceous Coccinellidae in biological control. Annual review of entomology. 43, 295-321 (1998).

66. Rocca, M., Rizzo, E. \& Greco, N. M. Larval interactions between two aphidophagous coccinellids in sweet pepper.Anais da Academia Brasileira de Ciências92 (2020).

67. Rocca, M., Rizzo, E., Greco, N. \& Sánchez, N. Intra-and interspecific interactions between anhidnnhannuc ladvhirdc the rnle nf nrey in predator coexistence. Entomologia Experimentalis et Loading [MathJax]/jax/output/CommonHTML/jax.js 
Applicata. 162, 284-292 (2017).

68. Aqueel, M. \& Leather, S. Nitrogen fertiliser affects the functional response and prey consumption of Harmonia axyridis (Coleoptera: Coccinellidae) feeding on cereal aphids. Annals of Applied Biology. 160, 6-15 (2012).

69. Koch, R. L., Hutchison, W. D., Venette, R. \& Heimpel, G. E. Susceptibility of immature monarch butterfly, Danaus plexippus (Lepidoptera: Nymphalidae: Danainae), to predation by Harmonia axyridis (Coleoptera: Coccinellidae). Biol. Control. 28, 265-270 (2003).

70. He, J., Ma, E., Shen, Y., Chen, W. \& Sun, X. Observations of the biological characteristics of Harmonia axyridis (Pallas)(Coleoptera: Coccinellidae). Journal of Shanghai Agricultural College. 12, 119-124 (1994).

71. Huang, Z. et al. Predation and functional response of the multi-coloured Asian ladybeetle Harmonia axyridis on the adult Asian citrus psyllid Diaphorina citri. Biocontrol Science and Technology. 29, 293-307 (2019).

72. Lee, J. H. \& Kang, T. J. Functional response of Harmonia axyridis (Pallas)(Coleoptera: Coccinellidae) to Aphis gossypii Glover (Homoptera: aphididae) in the laboratory. Biol. Control. 31, 306-310 (2004).

73. Xue, Y. et al. Predation by Coccinella septempunctata and Harmonia axyridis (Coleoptera: Coccinellidae) on Aphis glycines (Homoptera: Aphididae). Environ. Entomol. 38, 708-714 (2009).

74. Feng, Y., Zhou, Z. X., An, M. R., Yu, X. L. \& Liu, T. X. The effects of prey distribution and digestion on functional response of Harmonia axyridis (Coleoptera: Coccinellidae). Biol. Control. 124, 74-81 (2018).

75. Sandhi, R. K. \& Reddy, G. V. Biology, ecology, and management strategies for pea aphid (Hemiptera: Aphididae) in pulse crops. Journal of Integrated Pest Management. 11, 18 (2020).

76. Shah, F. M., Razaq, M., Ali, A., Han, P. \& Chen, J. Comparative role of neem seed extract, moringa leaf extract and imidacloprid in the management of wheat aphids in relation to yield losses in Pakistan. PloS one. 12, e0184639 (2017).

77. Shah, F. M. et al. Action threshold development in cabbage pest management using synthetic and botanical insecticides. Entomologia Generalis40 (2020).

78. Shah, F. M. et al. Field evaluation of synthetic and neem-derived alternative insecticides in developing action thresholds against cauliflower pests. Scientific reports. 9, 1-13 (2019).

79. Rix, R. \& Cutler, G. Low Doses of a Neonicotinoid Stimulate Reproduction in a Beneficial Predatory Insect. Journal of Economic Entomology. 113, 2179-2186 (2020).

80. Atlıhan, R. \& Güldal, H. Prey density-dependent feeding activity and life history of Scymnus subvillosus. Phytoparasitica. 37, 35-41 (2009).

81. Brown, J. H., Gillooly, J. F., Allen, A. P., Savage, V. M. \& West, G. B. Toward a metabolic theory of ecology. Ecology. 85, 1771-1789 (2004).

82. Vucic-Pestic, O., Ehnes, R. B., Rall, B. C. \& Brose, U. Warming up the system: higher predator feeding rates but lower energetic efficiencies. Glob. Change Biol. 17, 1301-1310 (2011).

Loading [MathJax]/jax/output/CommonHTML/jax.js

Page 23/30 
83. Lang, B., Rall, B. C. \& Brose, U. Warming effects on consumption and intraspecific interference competition depend on predator metabolism. Journal of Animal Ecology. 81, 516-523 (2012).

84. Wu, P., Zhang, J., Haseeb, M., Yan, S. \& Kanga, L. Functional responses and intraspecific competition in the ladybird Harmonia axyridis (Coleoptera: Coccinellidae) provided with Melanaphis sacchari (Homoptera: Aphididae) as prey. 115,232-241(2018).

85. Jafari, S., Fathipour, Y. \& Faraji, F. The influence of temperature on the functional response and prey consumption of Neoseiulus barkeri (Acari: Phytoseiidae) on Tetranychus urticae (Acari:

Tetranychidae). Journal of Entomological Society of Iran. 31, 39- 52 (2012).

86. Madbouni, M. A. Z., Samih, M. A., Namvar, P. \& Biondi, A. Temperature-dependent functional response of Nesidiocoris tenuis (Hemiptera: Miridae) to different densities of pupae of cotton whitefly, Bemisia tabaci (Hemiptera: Aleyrodidae). European journal of entomology. 114, 325 (2017).

87. Hodek, I., Honek, A. \& Van Emden, H. F. Ecology and behaviour of the ladybird beetles (Coccinellidae) (John Wiley \& Sons, 2012).

88. Li, Y. et al. The effect of different dietary sugars on the development and fecundity of Harmonia axyridis.Frontiers in Physiology11 (2020).

89. Zamani, A., Talebi, A., Fathipour, Y. \& Baniameri, V. Temperature-dependent functional response of two aphid parasitoids, Aphidius colemani and Aphidius matricariae (Hymenoptera: Aphidiidae), on the cotton aphid. Journal of Pest Science. 79, 183-188 (2006).

90. Sentis, A., Hemptinne, J. L. \& Brodeur, J. Using functional response modeling to investigate the effect of temperature on predator feeding rate and energetic efficiency. Oecologia. 169, 1117-1125 (2012).

91. Tully, T., Cassey, P. \& Ferrière, R. Functional response: rigorous estimation and sensitivity to genetic variation in prey. Oikos. 111, 479-487 (2005).

92. O'Neil, R. J. Comparison of laboratory and field measurements of the functional response of Podisus maculiventris (Heteroptera: Pentatomidae). Journal of the Kansas Entomological Society,148155(1989).

93. Wale, M., Jembere, B. \& Seyoum, E. Biology of the pea aphid, Acyrthosiphon pisum (Harris) (Homoptera: Aphididae) on cool-season legumes. International Journal of Tropical Insect Science. 20, 171-180 (2000).

94. de Oliveira Pimenta, I. C. et al. Effects of a Bt-based insecticide on the functional response of Ceraeochrysa cincta preying on Plutella xylostella. Ecotoxicology. 29, 856-865 (2020).

95. Hassanzadeh-Avval, M., Sadeghi-Namaghi, H. \& Fekrat, L. Factors influencing functional response, handling time and searching efficiency of Anthocoris minki Dohrn (Hem.: Anthocoridae) as predator of Psyllopsis repens Loginova (Hem.: Psyllidae). Phytoparasitica. 47, 341-350 (2019).

96. Banihashemi, A. S., Seraj, A. A., Yarahmadi, F. \& Rajabpour, A. Effect of host plants on predation, prey preference and switching behaviour of Orius albidipennis on Bemisia tabaci and Tetranychus turkestani. International Journal of Tropical Insect Science. 37, 176-182 (2017).

97. Abbott, W. S. A method of computing the effectiveness of an insecticide. J. econ. Entomol. 18, 265- 
98. Rogers, D. Random search and insect population models. The Journal of Animal Ecology,369383(1972).

99. Pritchard, D. W., Paterson, R. \& Bovy, H. C. \& Barrios-O'Neill, D. Frair: an R package for fitting and comparing consumer functional responses. Methods in Ecology and Evolution. 8, 1528-1534 (2017).

100. R Core Team. R: A language and environment for statistical computing. R Foundation for Statistical Computing 2014, Vienna, Austria (2014).

101. Hassell, M. The spatial and temporal dynamics of host-parasitoid interactions (Oxford University Press, 2000).

\section{Figures}
(a) Temperature $\left({ }^{\circ} \mathrm{C}\right)$
(b) Initial prey density
(c) Temperature by density
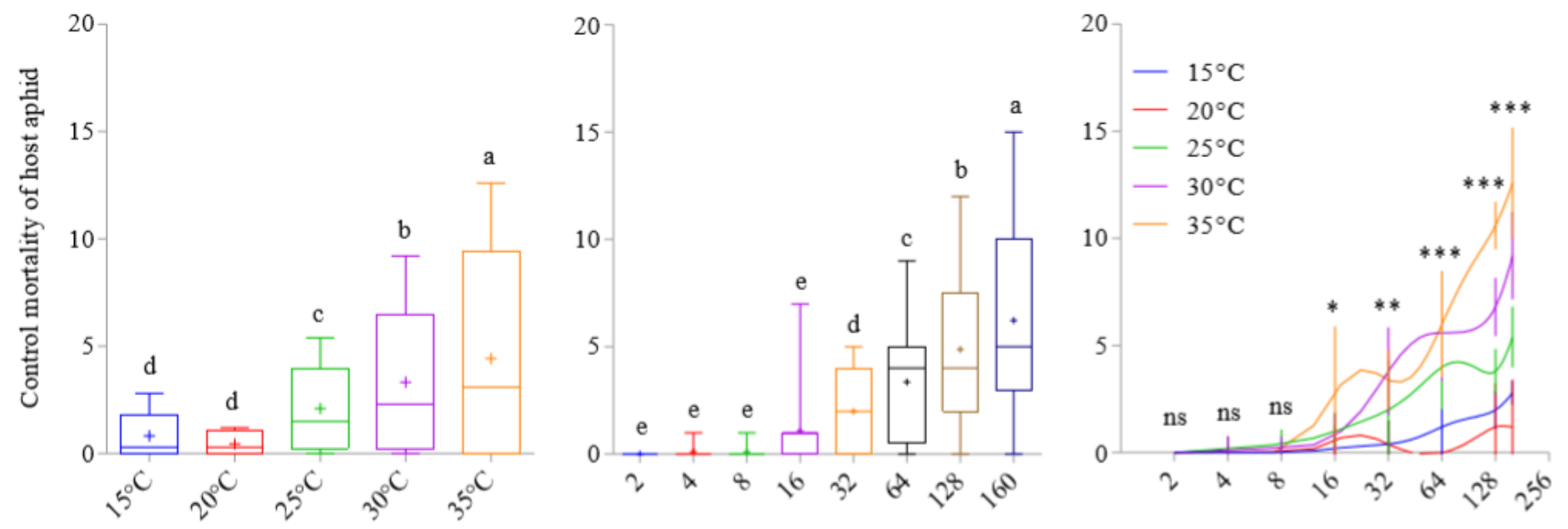

\section{Figure 1}

Control mortality of host aphid across temperatures and initial prey densities. Box plots showing the range of data (lower and upper quartiles, and extreme values), median, and mean (symbols). Box plots as in panels $a, b$, labelled with the different letter are showing significant differences among group means ( $P$ $<0.05$; Tukey's HSD test), whereas panel c showing significant difference $(P<0.05)$ based on nonoverlapping $95 \% \mathrm{Cl}$ of difference. ${ }^{*}, * *$, and ${ }^{* *}$ denote significance at $0.05,0.01$, and 0.001 levels, respectively. 

(a) Temperature $\left({ }^{\circ} \mathrm{C}\right)$
(b) Stage
(c) Initial prey density
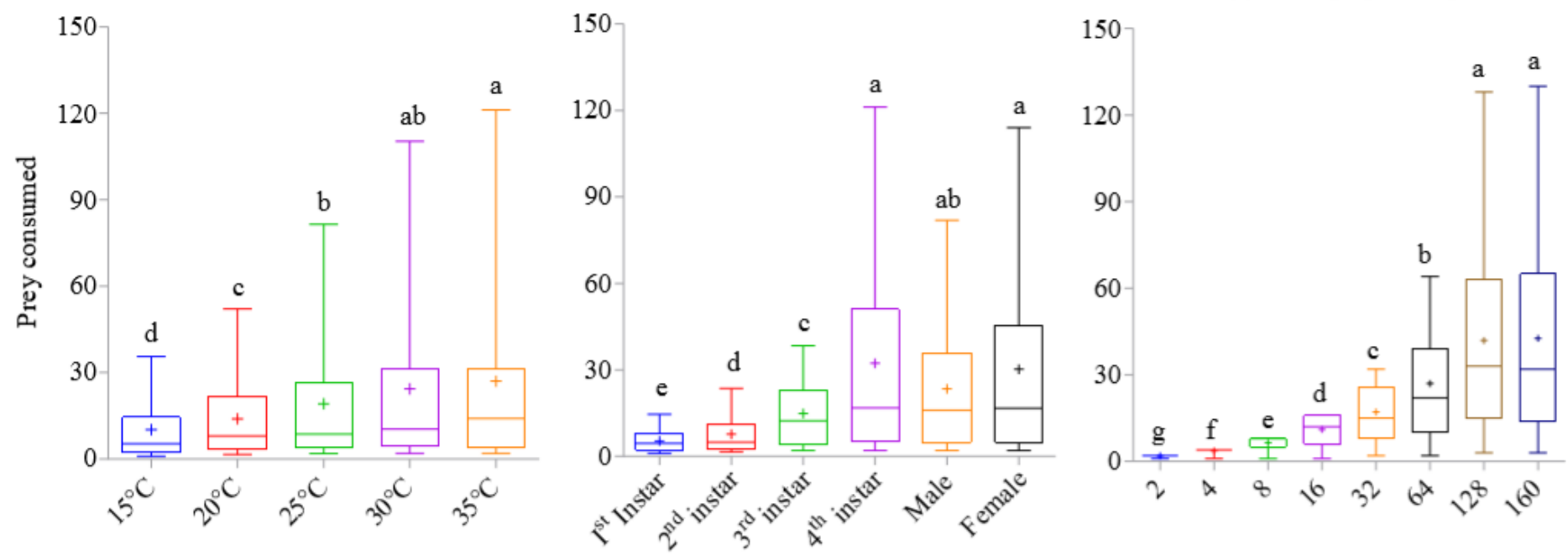

\section{Figure 2}

Mean number of prey consumed by Harmonia axyridis across temperatures, predatory stages and initial prey densities. Box plots showing the range of data (lower and upper quartiles, and extreme values), median, and mean (symbols). Box plots labelled with the different letter are showing significant differences among group means according to Wald chi square test with $95 \% \mathrm{Cl}$ of difference. 
(a) $\mathrm{I}^{\text {st }}$ instar
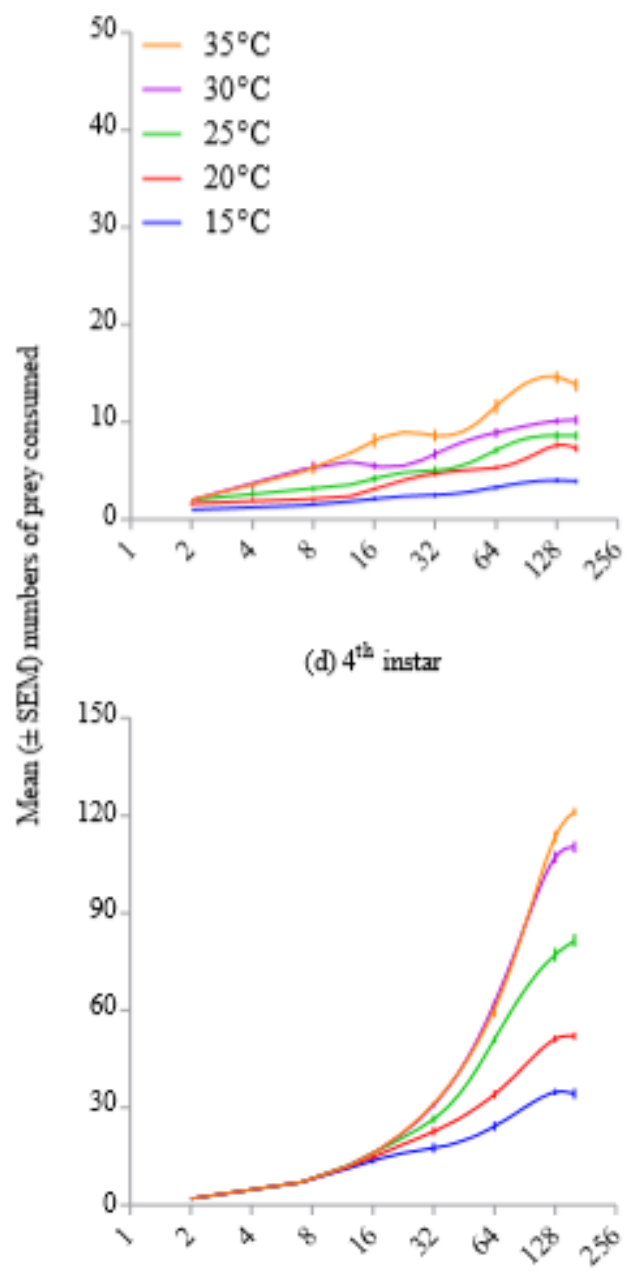

(b) $2^{\text {nd }}$ instar

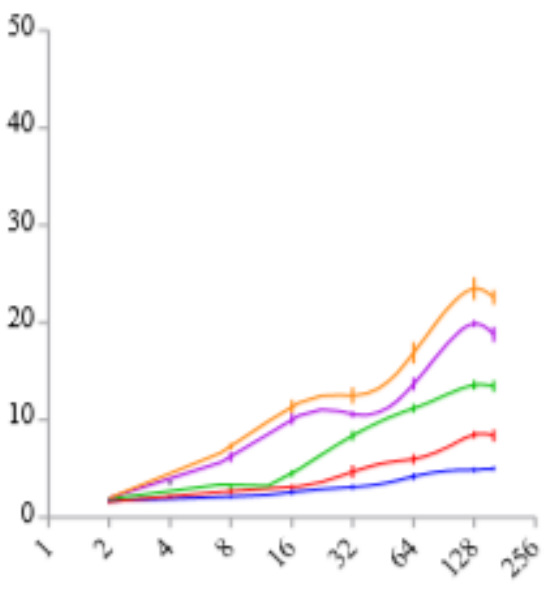

(e) Male

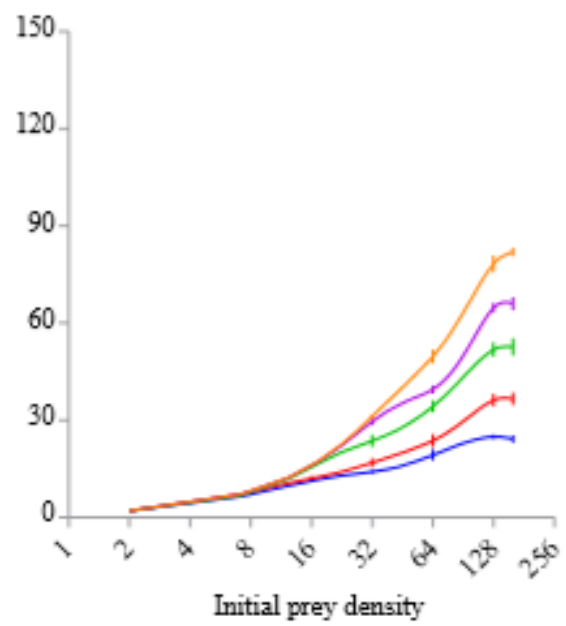

(c) $3^{\text {nd }}$ instar

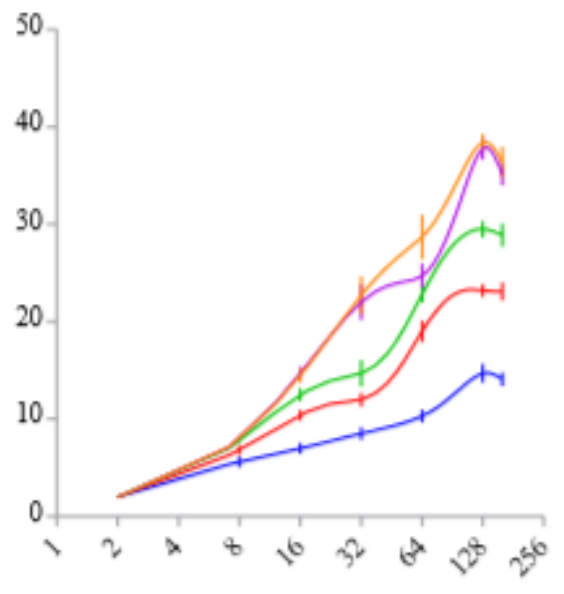

(f) Female

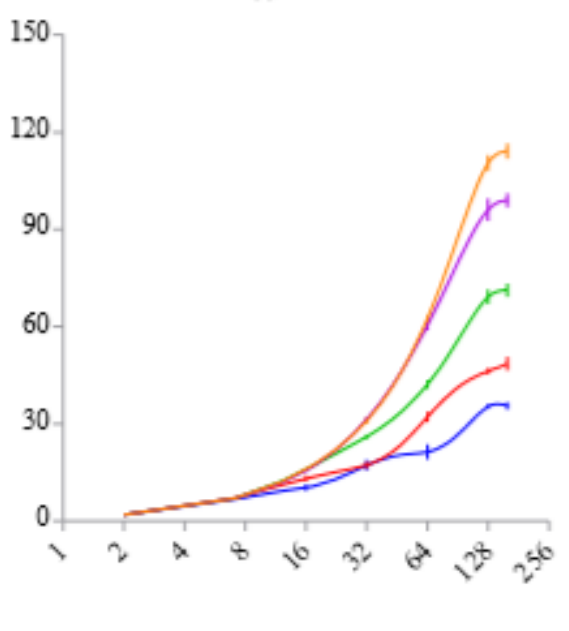

\section{Figure 3}

Mean ( \pm SEM) numbers of prey consumed by Harmonia axyridis across temperatures with respect to the predator growth stages and initial prey densities. 
(a) $15^{\circ} \mathrm{C}$

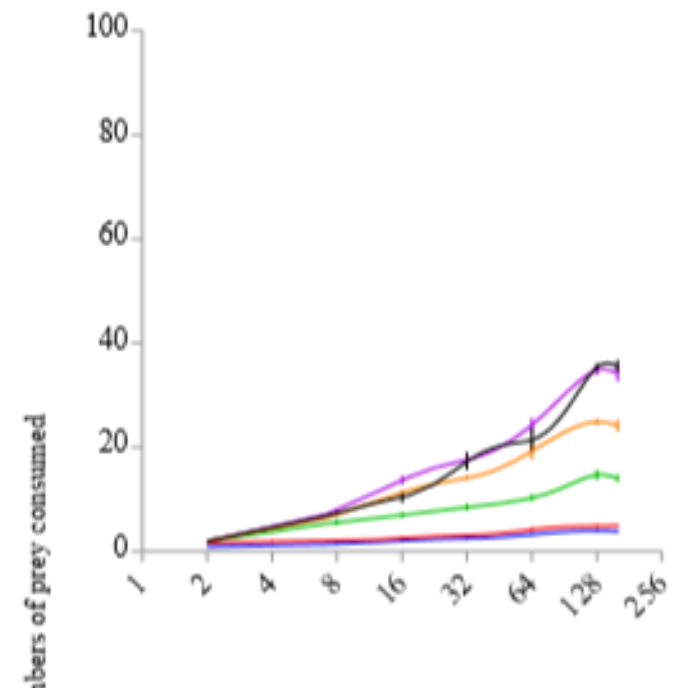

(d) $30^{\circ} \mathrm{C}$

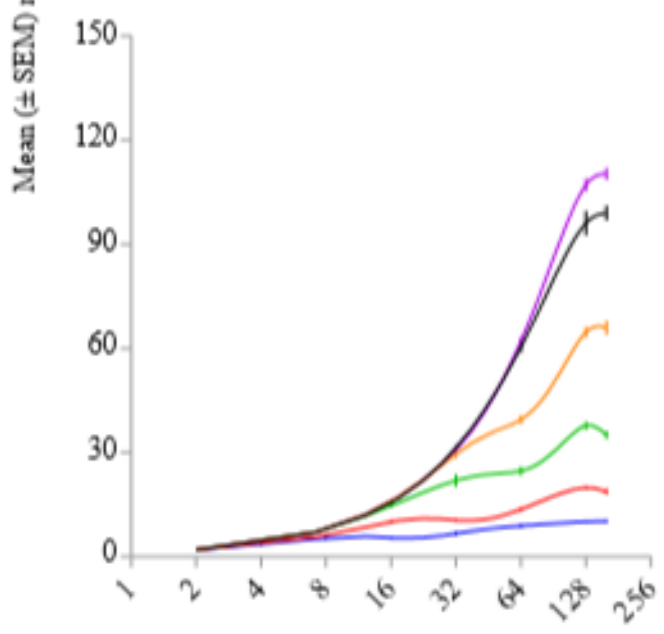

(b) $20^{\circ} \mathrm{C}$

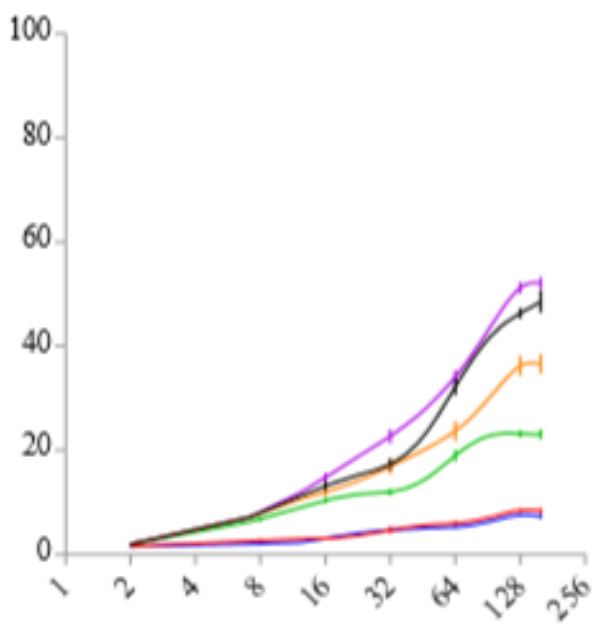

(e) $35^{\circ} \mathrm{C}$

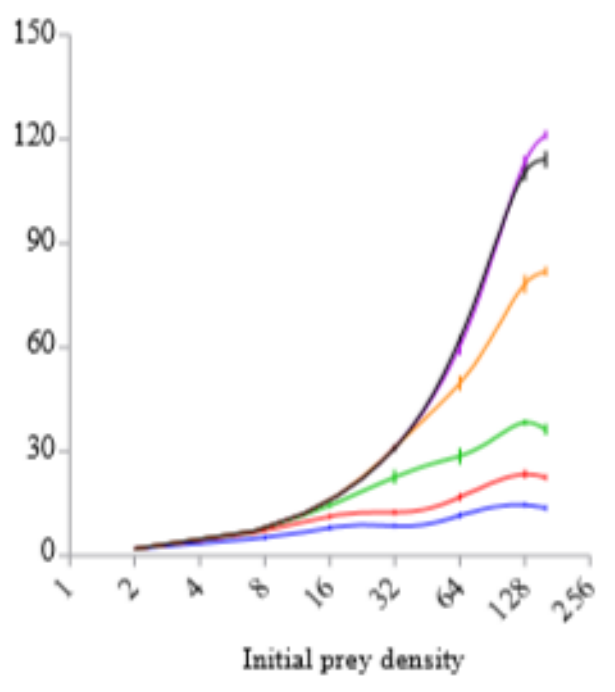

(c) $25^{\circ} \mathrm{C}$
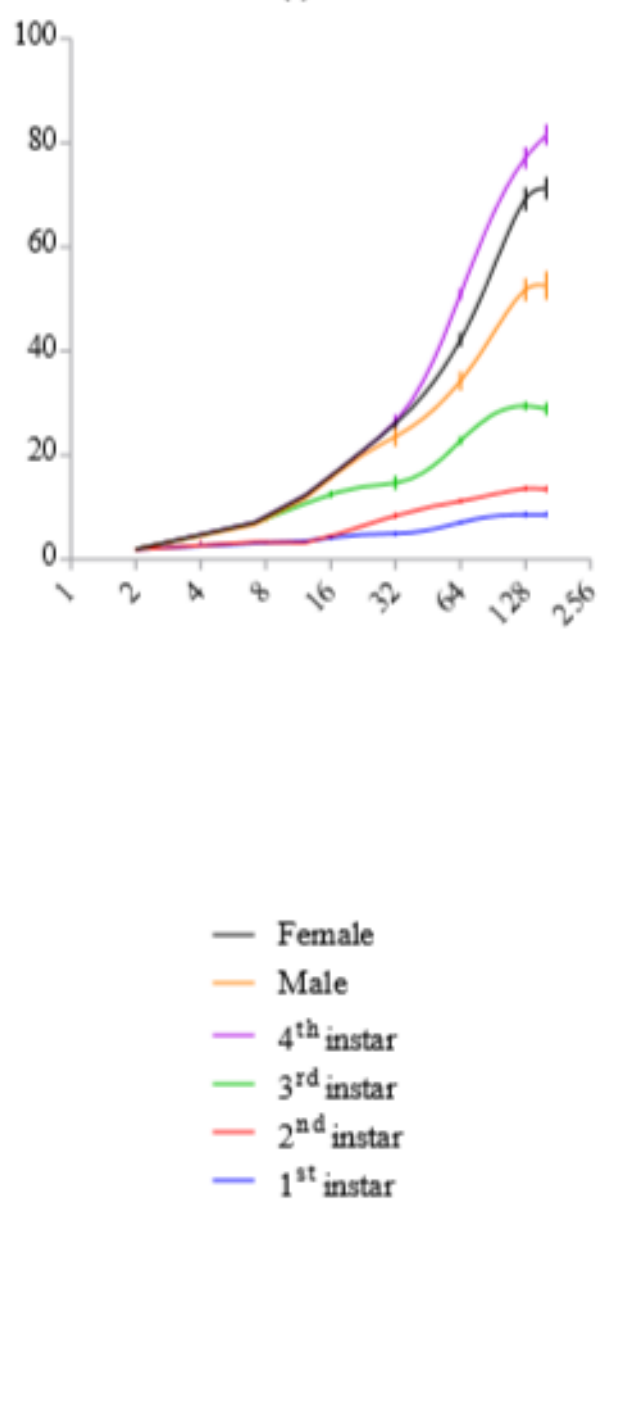

\section{Figure 4}

Mean ( \pm SEM) numbers of prey consumed by Harmonia axyridis across predatory stages with respect to the thermal conditions imposed and initial prey densities. 
(a) $I^{\text {st }}$ instar

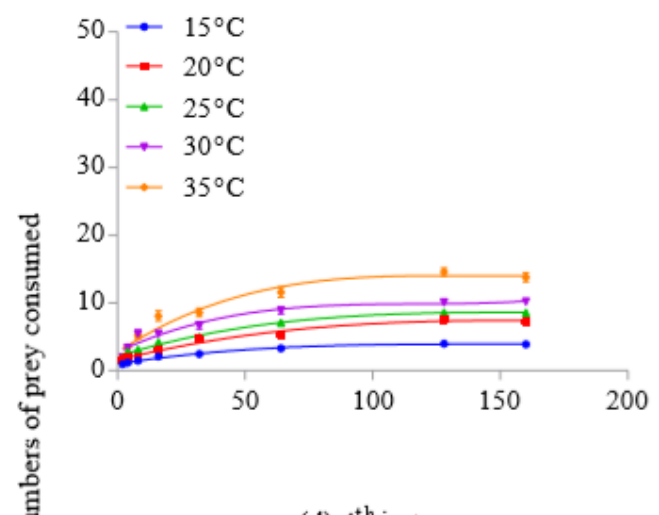

(d) $4^{\text {th }}$ instar

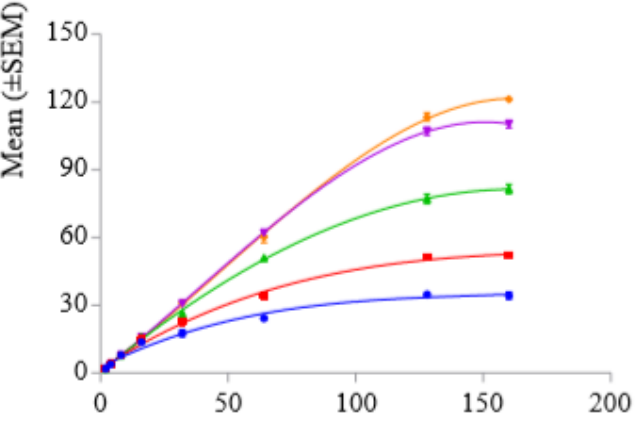

(b) $2^{\text {nd }}$ instar

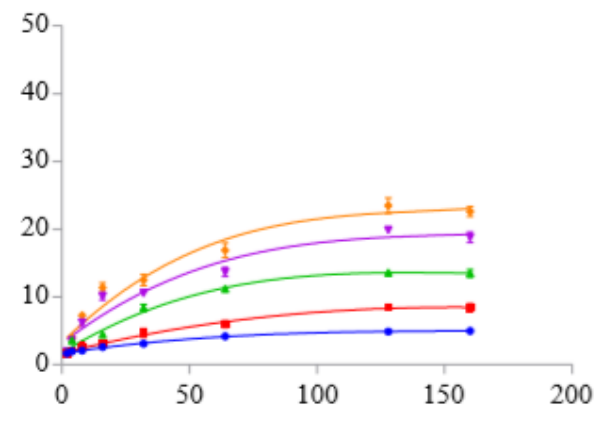

(e) Male

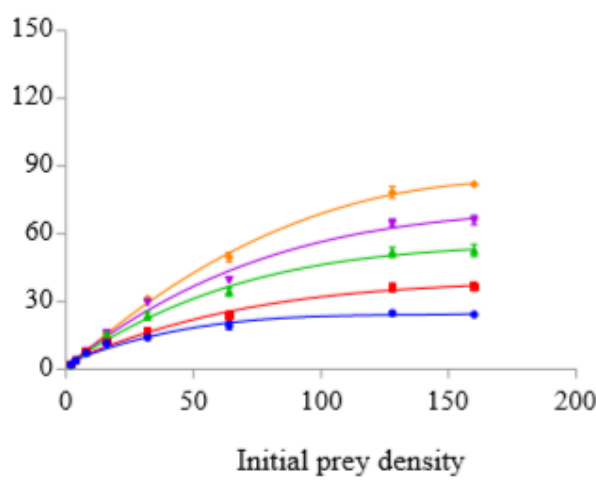

(c) $3^{\text {rd }}$ instar

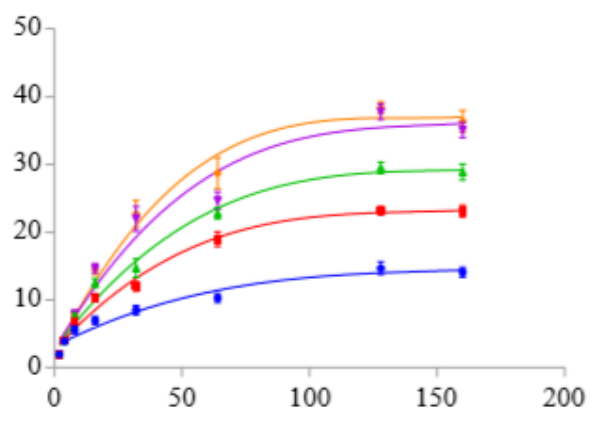

(f) Female

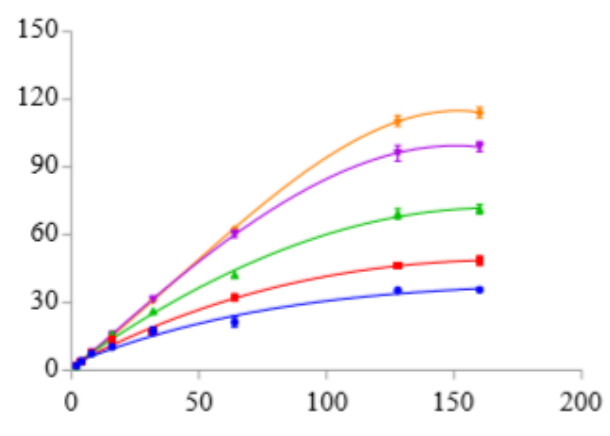

\section{Figure 5}

Type II functional response curves fitted by Roger's random predator equation of Harmonia axyridis across temperatures. 
(a) $\mathrm{P}^{\text {st }}$ instar

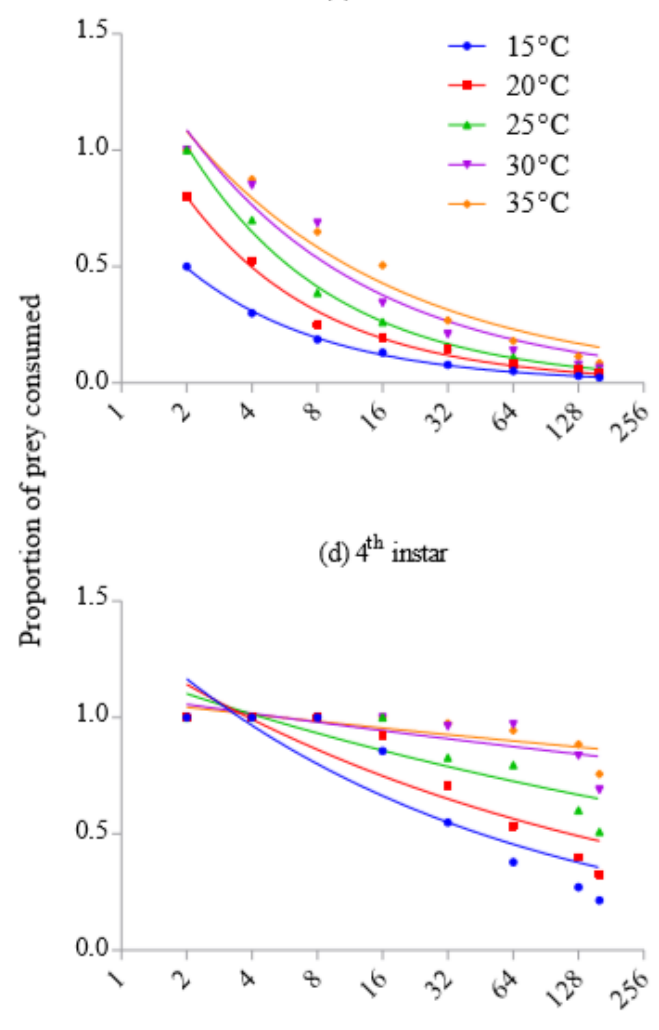

(b) $2^{\text {nd }}$ instar

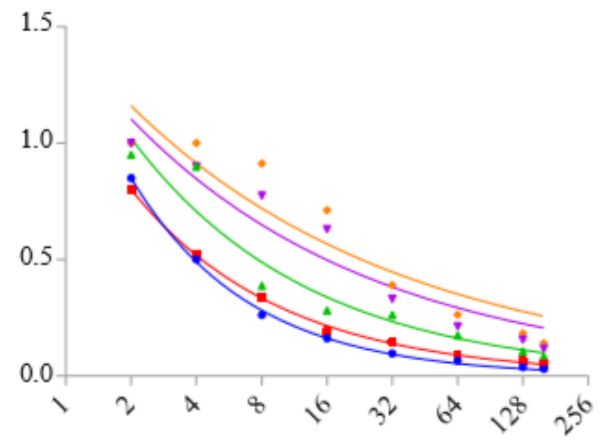

(e) Male

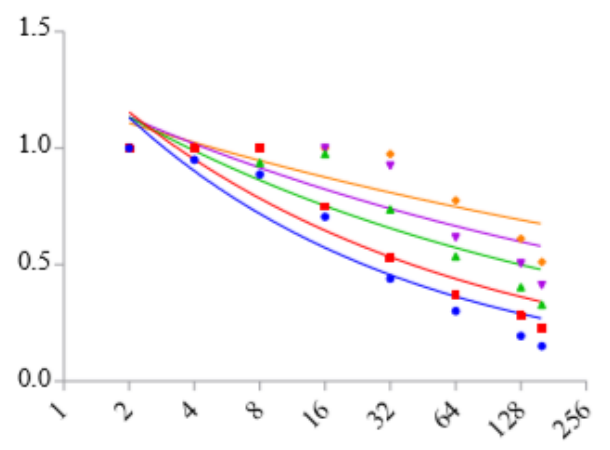

Initial prey density (c) $3^{\text {rd }}$ instar

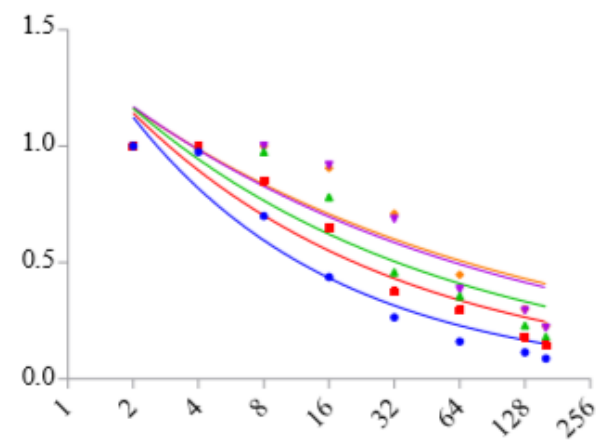

(f) Female

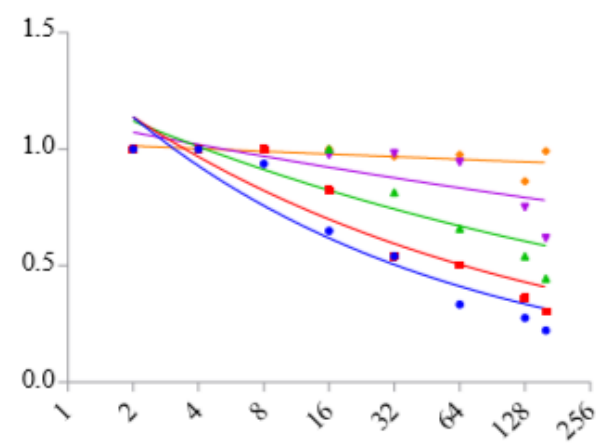

\section{Figure 6}

Proportion of prey consumed by Harmonia axyridis across temperatures and initial prey densities.
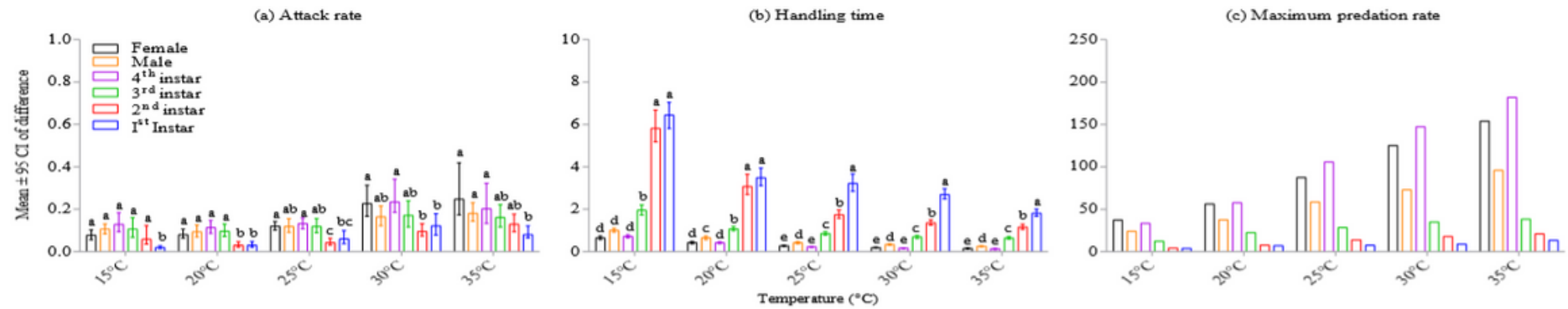

\section{Figure 7}

Functional response parameters of Harmonia axyridis across temperatures, resulting from bootstrapped functional response parameters. Bars are estimated means with $95 \mathrm{Cl}$ of difference. Different letters above the bars within each temperature are showing significant difference $(P<0.05)$ among various growth stages. 